\title{
Chapter 35 \\ Mathematical Morphology \\ in Geosciences and GISci: \\ An Illustrative Review
}

\author{
B. S. Daya Sagar
}

\begin{abstract}
Georges Matheron and Jean Serra of the Centre of Mathematical Morphology, Fontainebleau founded Mathematical Morphology (MM). Since the birth of MM in the mid 1960s, its applications in a wide ranging disciplines have illustrated that intuitive researchers can find varied application-domains to extend the applications of MM. This chapter provides a concise review of application of Mathematical Morphology in Geosciences and Geographical Information Science (GISci). The motivation for this chapter stems from the fact that Mathematical Morphology is one of the better choices to deal with highly intertwined topics such as retrieval, analysis, reasoning, and simulation and modeling of terrestrial phenomena and processes. This chapter provides an illustrative review of various studies carried out by the author over a period of 25 years - related to applications of Mathematical Morphology and Fractal Geometry-in the contexts of Geosciences and Geographical Information Science (GISci). However, the reader is encouraged to refer to the cited publications to gather more details on the review provided in an abstract manner.
\end{abstract}

\subsection{Introduction}

A basic understanding of many geoscientific and geoengineering challenges across multiple spatial and/or temporal scales of terrestrial phenomena and processes is among the greatest of challenges facing contemporary sciences and engineering. Many space-time models explaining phenomena and processes of terrestrial relevance were descriptive in nature. Earlier, several toy models were developed via classical mathematics to explain possible phases in dynamical behaviors of complex systems. With the advent of computers with powerful graphics facilities, about three decades ago the interplay between numerical methods (generated via classical

\section{B. S. Daya Sagar (四)}

Systems Science and Informatics Unit, Indian Statistical Institute-Bangalore Centre, 8th Mile, Mysore Road, RVCE PO, Bengaluru 560059, India

e-mail: bsdsagar@isibang.ac.in

B. S. Daya Sagar et al. (eds.), Handbook of Mathematical Geosciences, 
equations explaining the behaviors of dynamical systems) and graphics was shown to exist. That progress provided the initial impetus to visualize the systems' spatial and/or temporal behaviors that exhibit simple to complex patterns on graphical screens. One of the efficient ways of understanding the dynamical behavior of many complex systems of nature, society and science is possible through data acquired at multiple spatial and temporal scales. Data related to terrestrial (geophysical) phenomena at spatial and temporal intervals are available in numerous formats. The utility and application of such data could be substantially enhanced through related technologies documented in edited volumes and monographs of the recent past (Sagar 2001a, b, c, d, 2005a, b, 2009, 2013; Sagar and Rao 2003; Sagar et al. 2004; Sagar and Bruce 2005; Sagar and Serra 2010; Najman et al. 2012).

To understand the dynamical behavior of a phenomenon or a process, development of a good spatiotemporal model is essential. To develop a good spatiotemporal model, well-analyzed and well-reasoned information that could be extracted/retrieved from spatial and/or temporal data are important ingredients. Figure 35.1 shows a schematic illustrating the key links between the various phases where the involvement of Mathematical Morphology becomes obvious from the studies to be shown later in the chapter.

Mathematical Morphology_founded by Georges Matheron (1975) and Jean Serra (1982) has shown great impact in various fields including Geosciences and GISci-is one of the better choices to deal with all these key aspects mentioned. Mathematical morphology was founded by Georges Matheron (Agterberg 2001, 2004; Serra 1982, 1988). There are numerous representative publications related to mathematical morphology, to name a few: Serra (1982, 1988), Sternberg (1986), Beucher (1990, 1999), Soille (2003), Najman and Talbot (2010), Sagar (2013). Most notably, the comment on the issue of "What do Mathematical Geoscientists

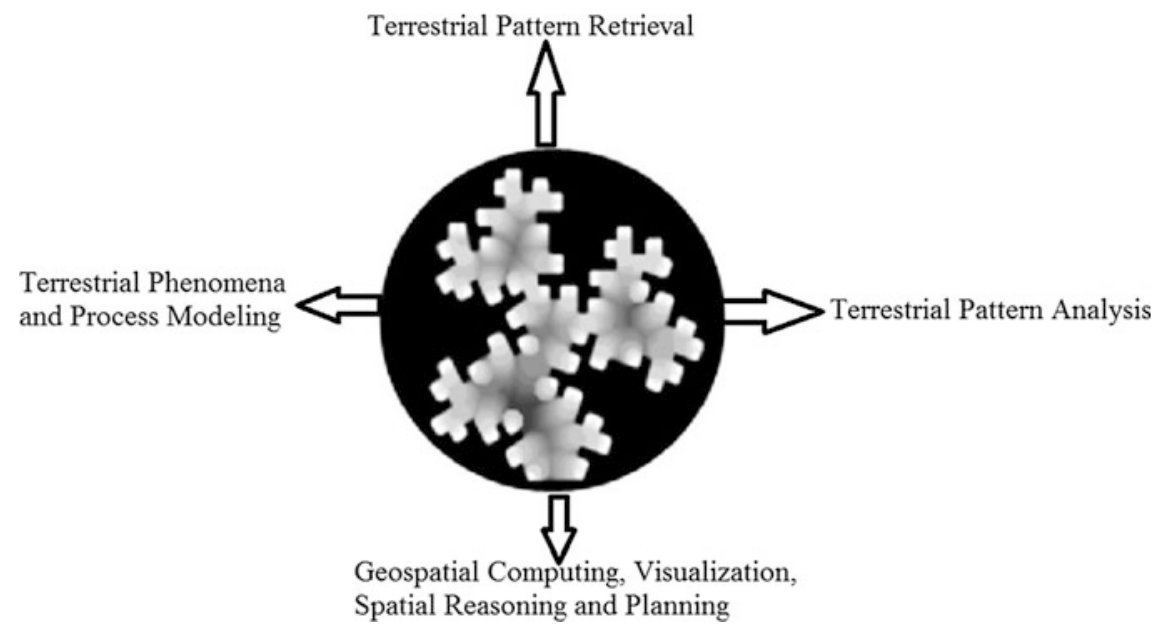

Fig. 35.1 Mathematical morphology applications in several phases of studies of relevance to geosciences and geographical information science 
Table 35.1 Successful applications of MM transformations in geosciences, geomorphology, GISci-major references

\begin{tabular}{l|l|l}
\hline Morphological operator & Application domain & Major references \\
\hline $\begin{array}{l}\text { Binary and grayscale } \\
\text { morphological erosion, } \\
\text { dilation, opening, closing, } \\
\text { multiscale morphological } \\
\text { operations }\end{array}$ & $\begin{array}{l}\text { Petrology, GISci, } \\
\text { geosciences, remote } \\
\text { sensing }\end{array}$ & $\begin{array}{l}\text { Serra (1982), Sagar (2013), } \\
\text { Brunet and Sills (2017), } \\
\text { Beucher (1990, 1999) }\end{array}$ \\
\hline $\begin{array}{l}\text { Geodesic morphological } \\
\text { operations }\end{array}$ & $\begin{array}{l}\text { Remote sensing, GISci, } \\
\text { geography, petrology }\end{array}$ & $\begin{array}{l}\text { Lantuejoul (1978), Lantuejoul } \\
\text { and Beucher (1981), Sagar and } \\
\text { Lim (2008a, b), Challa et al. } \\
\text { (2016) }\end{array}$ \\
\hline $\begin{array}{l}\text { Hit-or-miss transformation } \\
\text { Morphological thinning, } \\
\text { thickening, pruning }\end{array}$ & $\begin{array}{l}\text { Geomorphology, } \\
\text { hydrology }\end{array}$ & $\begin{array}{l}\text { Serra (1982), Tay et al. (2005a, } \\
\text { bydrology, cartography }\end{array}$ \\
\hline $\begin{array}{l}\text { Morphological } \\
\text { skeletonization }\end{array}$ & $\begin{array}{l}\text { Soille (2003), Sagar (2013) } \\
\text { geomorphology }\end{array}$ & $\begin{array}{l}\text { Sagar et al. (2000, 2003a, b), } \\
\text { Soille (2003) }\end{array}$ \\
\hline $\begin{array}{l}\text { Skeletonization by zones of } \\
\text { influence and weighted } \\
\text { skeletonization by zones of } \\
\text { Influence }\end{array}$ & $\begin{array}{l}\text { Cartography, hydrology, } \\
\text { geomorphology }\end{array}$ & $\begin{array}{l}\text { Beucher (1990), Rajashekara } \\
\text { et al. (2012), Sagar (2014a, b) }\end{array}$ \\
\hline $\begin{array}{l}\text { Granulometries and } \\
\text { anti-granulometries }\end{array}$ & $\begin{array}{l}\text { Petrology, } \\
\text { geomorphology, } \\
\text { hydrology }\end{array}$ & $\begin{array}{l}\text { Serra (1982), Maragos (1989), } \\
\text { Sagar (2013), Tay et al. } \\
\text { (2005a, b, c 2007), Vardhan } \\
\text { et al. (2013) }\end{array}$ \\
\hline $\begin{array}{l}\text { Morphological distances, } \\
\text { hausdorff dilation (erosion) } \\
\text { distances }\end{array}$ & $\begin{array}{l}\text { GiSci, limnology, } \\
\text { biogeography, spatial } \\
\text { planning }\end{array}$ & $\begin{array}{l}\text { Serra (1988), Sagar (2010, } \\
\text { and extrapolations } \\
\text { b) Sagar and Lim (2015a, }\end{array}$ \\
\hline $\begin{array}{l}\text { Watershed transformation } \\
\text { science, geology, remote } \\
\text { sensing, cartography }\end{array}$ & $\begin{array}{l}\text { Sagar (2010) Brunet and Sills } \\
\text { (2017), Rajashekara et al. } \\
\text { (2012), Sagar (2014a, b), Sagar } \\
\text { and Lim (2015a, b) }\end{array}$ \\
\hline $\begin{array}{l}\text { Hydrology, remote } \\
\text { sensing, mapping, } \\
\text { borehole studies, seismic } \\
\text { data processing }\end{array}$ & $\begin{array}{l}\text { Meyer (1980), Beucher and } \\
\text { (1992), Sagar (2007) }\end{array}$ \\
\hline
\end{tabular}

Do?" made by Harbaugh (2014) includes the importance of mathematical morphology of geological features in making predictions. In this chapter we outline the successful applications of the most important concepts of mathematical morphology (Table 35.1) in the context of geosciences and Geographical Information Science (GISci).

While perceiving the terrestrial surfaces including geophysical and geomorphic basins (e.g. using Digital Elevation Models, Digital Bathymetric Models, cloud fields, microscale rock porous media etc.) as functions, planar forms (e.g. topographic depressions, water bodies, and threshold elevation regions, hillslopes) as sets, and abstract structures (e.g. networks and watershed boundaries) as skeletons, 
we make attempts to unravel key links for better understanding spatiotemporal behaviors of several terrestrial and/or spatial phenomena and processes between the following coherent aspects: (i) terrestrial pattern retrieval, (Sect. 35.2) (ii) terrestrial pattern analysis, (Sect. 35.3) (iii) simulation and modeling, (Sect. 35.4) and (iv) geocomputing, visualization, spatial reasoning and planning (Sect. 35.5).

\subsection{Terrestrial Pattern Retrieval}

Retrieving relevant information from precisely acquired spatial-temporal data of varied types about a specific complex system is a basic prerequisite to understand the spatial-temporal behavior of a system. Retrieval of information from a available spatiotemporal data acquired from a wide range of sources and a variety of formats, opens new horizons to the spatial statistical and geoscience communities. We have developed original spatial algorithms based on non-linear morphological transformations for retrieval of unique geophysical networks, mountain objects, segmentation of various geophysical objects, and pairing the geophysical spatial fields based on certain similarities (Sagar et al. 2000, 2003a, b; Sagar and Chockalingam 2004; Sathymoorthy et al. 2007; Chockalingam and Sagar 2003; Lim and Sagar 2008a, b; Lim et al. 2009, 2011; Sagar and Lim 2015a, b; Danda et al. 2016).

\subsubsection{Mathematical Morphology in Extraction of Unique Topological Networks}

In contrast to other recent works, which have focused on extraction of channel networks via algorithms that fail to precisely extract networks from non-hilly regions (e.g. tidal regions), the algorithms we proposed can be generalized for application to both hilly (e.g. fluvial) and non-hilly (e.g. tidal) terrains, and also pore connectivity networks. These algorithms concerning the framework to extract multiscale geomorphologic networks via systematically decomposing elevation surfaces and/or decomposed threshold elevation regions into their abstract structures lead to valley and ridge connectivity networks. We proposed a framework to first decompose a binary fractal basin into fractal DEM from which two unique topological connectivity networks are extracted. These networks facilitate to segment Fractal-DEM (Fig. 35.2a) into sub-basins ranging from first to highest order (Fig. 35.2c). Results derived from a synthetic DEM (Fig. 35.2a) by applying one of these algorithms include unique topological connectivity networks similar to valley and ridge connectivity networks (Fig. 35.2b) and the hierarchically partitioned watersheds (Fig. 35.2c). We demonstrated the superiority of these stable algorithms which can be generalized to terrestrial surfaces of both fluvial and tidal types. This 
(a)

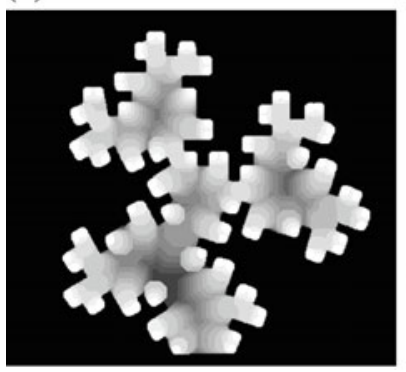

(b)

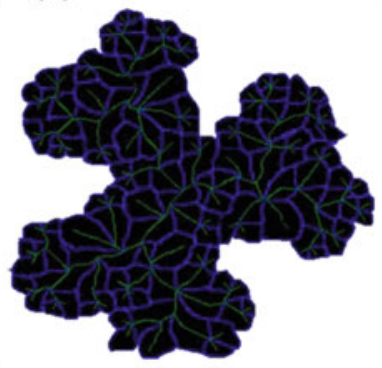

(c)

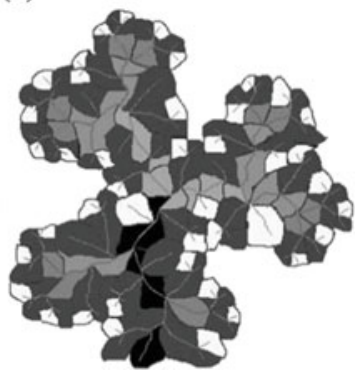

Fig. 35.2 a simulated fractal DEM achieved through morphological decomposition procedure, b loop-like ridge connectivity and loopless channel connectivity networks, and c subbasins

work helps to solve basic problems that algorithms meant for extraction of unique terrestrial connectivity networks have faced for over three decades.

\subsubsection{Retrieval of Morphologically Significant Regions}

Algorithms meant for morphological segmentation were demonstrated on a DEM, and mapped the physiographic features such as mountains, basins, and piedmont slopes from DEM (Fig. 35.3a); and the results are compared with that of other popular approaches (Fig. 35.3b).

Further, multiscale morphological opening was employed to segment binary fractal basins (Fig. 35.4a-c) that mimic geophysical basins, and cloud fields

(a)

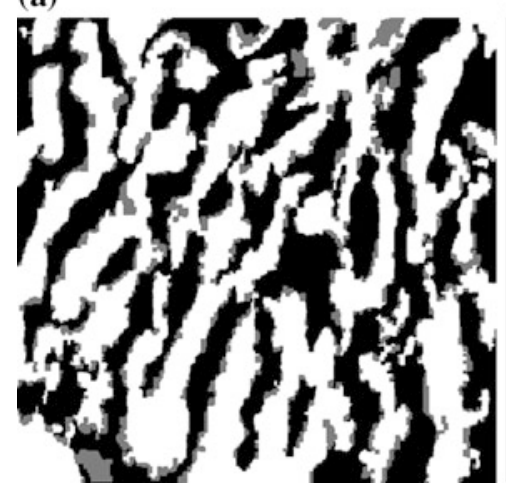

(b)

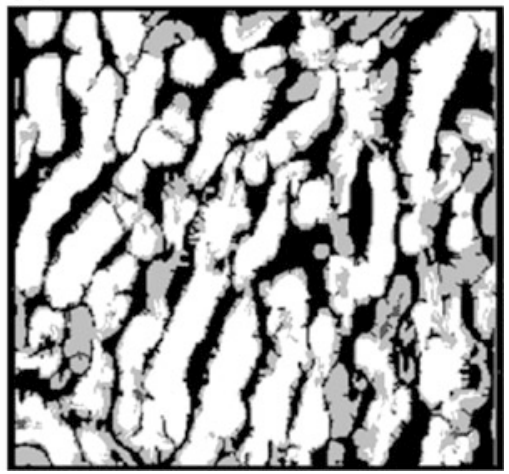

Fig. 35.3 Mountain pixels are the pixels in white, the piedmont pixels are the pixels in gray, and the basin pixels are the pixels in black. a The results obtained using the newly developed algorithm. b The results obtained in Miliaresis and Argialas (1999). (From Sathymoorthy et al. 2007) 
(a)

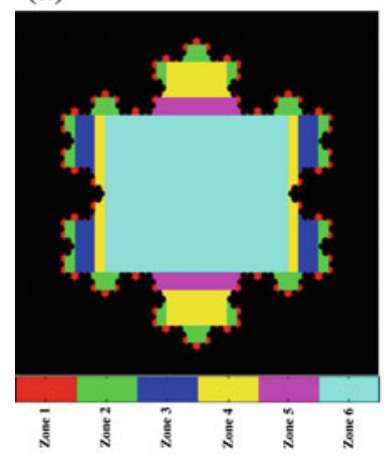

(d)

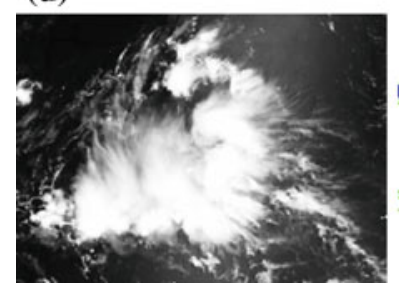

(b)

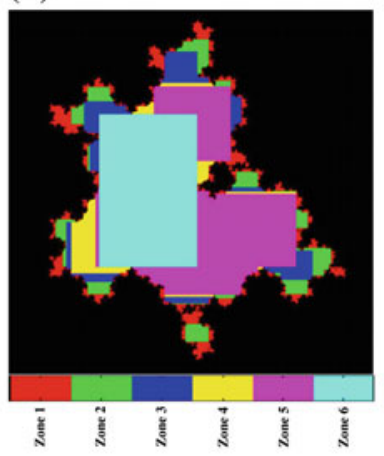

(e)

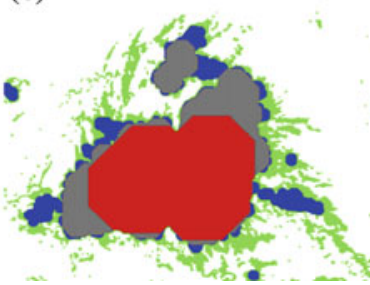

(c)

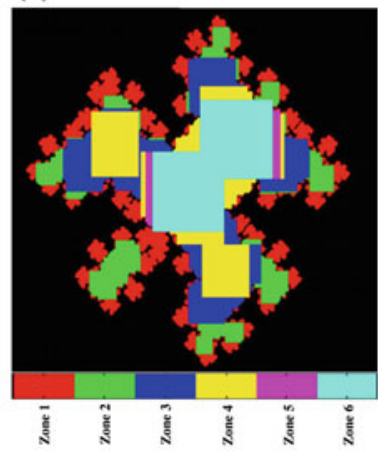

(f)

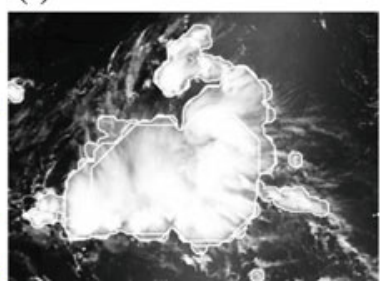

Fig. 35.4 Morphologically significant zones decomposed from a Koch triadic fractal island, b Random Koch triadic fractal island, c Random Koch quadric fractal island, d Isolated Moderate Resolution Imaging Spectroradiometer (MODIS) cloud (cloud-1), e Color-coded binarized (by choosing threshold gray level value 128) cloud-1 images at three threshold-opening cycles superimposed on binarized original cloud-1 color-coded with green, and $\mathbf{f}$ boundaries of 12th, 32 nd, and 100th opened cloud-1 images and thresholded original cloud-1 superimposed on the original cloud image

isolated from MODIS data into topologically prominent regions (Fig. 35.4d-f). We proposed granulometry-based segmentation of geophysical fields (e.g. DEMs, clouds, etc.) with demonstration on binary fractals of deterministic and random types (Fig. 35.4a-c), and on cloud fields (Fig. 35.4d-f) that have different compaction properties with varied cloud properties.

The approach based on computation of complexity measures of morphologically significant zones decomposed from binary fractal sets via multiscale convexity analysis - which can be implemented on several geophysical and geomorphologic fields (e.g. DEMs, clouds, binary fractals etc.) to segment them into regions of varied topological significance-has been demonstrated on cloud fields derived from MODIS data to better segment the regions within the cloud fields that have different compaction properties with varied cloud properties. This approach of fundamental importance can be extended to several geophysical and geomorphologic fields to segment them into regions of varied topological significance. 

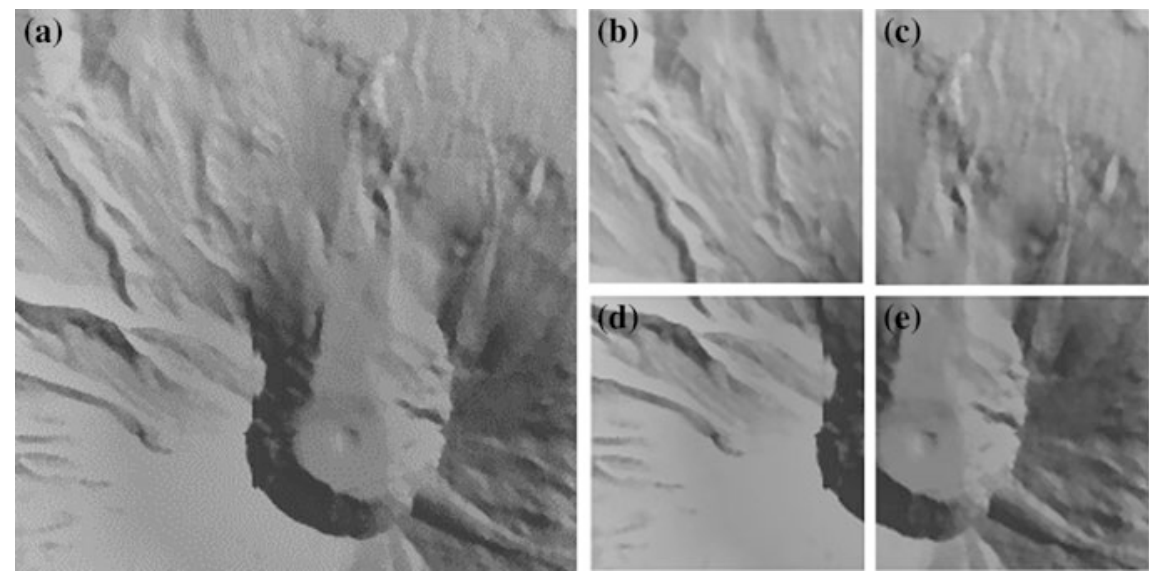

Fig. 35.5 a Digital Elevation Model of size $256 \times 256$ pixels depicting Mount St Helens, b-e four quadrants of size $128 \times 128$ pixels partitioned from DEM (Fig. 35.5a) include top-left $\left(f^{1}\right)$, top-right $\left(f^{2}\right)$, bottom-left $\left(f^{3}\right)$, and bottom-right $\left(f^{4}\right)$ portions

\subsubsection{Ranking of Best Pairs of Spatial Fields}

A new metric to quantify the degree of similarity between any two given spatial fields is proposed (Sagar and Lim 2015a, b). This metric based on morphological operations can be used for image classification, in particular hyperspectral image classification, to derive best pair(s) of spatial fields from among a large number of spatial fields available in a database. In this proposed approach to compute the ranks for every possible pair of spatial fields (grayscale images) in a database, the two major computations involved include (i) estimation of grayscale morphological distance between the source and target spatial fields, and (ii) the ratios between the areas of infima and suprema of source and target spatial fields. Using this approach, four spatial elevation fields (Fig. 35.5b-e), in other words four quadrants partitioned from Fig. 35.5a could be paired into best pair (Fig. 35.6a), medium best pair (Fig. 35.6b), and the least best pair (Fig. 35.6c).

(a)

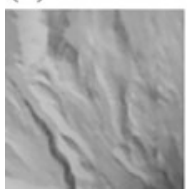

(b)

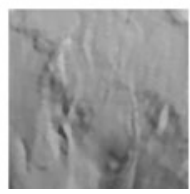

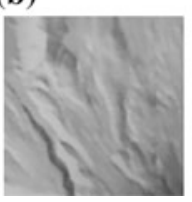

(c)

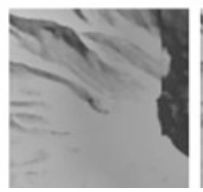

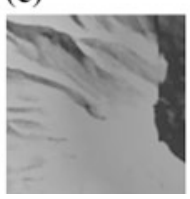

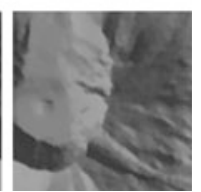

Fig. 35.6 Three best ranked pairs of spatial elevation fields shown in Fig. 35.5b-e a $\left(f^{1}, f^{2}\right)$, $\mathbf{b}\left(f^{1}, f^{3}\right)$, and $\mathbf{c}\left(f^{3}, f^{4}\right)$ 


\subsection{Terrestrial Pattern Analysis}

Quantitative analyses of terrestrial phenomena and processes is one of the innovative new directions of geoscientific research. Analysis of terrestrial patterns - that include water bodies, valley and ridge connectivity networks, watersheds, hillslopes, mountain objects, elevation fields - at various spatial and temporal scales is an important aspect to better understand the dynamical behaviors of various terrestrial processes and surfaces. Over the decades, various quantitative approaches have been developed and successfully demonstrated. Some of these approaches include morphometric analysis of river networks, hypsometry, allometry, and granulometric analyses, and geodesic spectrum based analysis. In this section, we show some results through illustrations arrived at via applications of mathematical morphology in (i) morphometric and allometric analyses of river networks and water bodies and their corresponding zones of influence, (ii) deriving scale-invariant but shape-dependant power laws, (iii) deriving basin-specific geodesic spectrum, and (iv) DEM analysis.

\subsubsection{Morphometry and Allometry of Networks}

Towards analyzing terrestrial surfaces we have shown unique ways to quantitatively characterize the spatiotemporal terrestrial complexity via scale-invariant measures that explain the commonly sharing physical mechanisms involved in terrestrial phenomena and processes. These contributions (Sagar and Rao 1995a, b, c, d; Sagar 1996, 1999a 2000a, b, 2001a, b, c, d 2007; Sagar et al. 1998a, b, 1999; Sagar and Tien 2004; Chockalingam and Sagar 2005; Tay et al. 2005a, b, c) highlighted the evidence of self-organization via scaling laws-in networks, hierarchically decomposed subwatersheds, and water bodies and their zones of influence, which evidently belong to different universality classes-which possess excellent agreement with geomorphologic laws such as Horton's Laws, Hurst exponents, Hack's exponent, and other power-laws given in non-geoscientific contexts. A host of allometric power-law relationships were derived that were in good accord with other established network models and real networks (Figs. 35.7, 35.8 and 35.9).

\subsubsection{Allometry of Water Bodies and Their Zones of Influence}

Topologically, water bodies (Fig. 35.10a) are the first level topographic regions that get flooded, and as the flood level gets higher, adjacent water bodies merge. The looplike network that forms along all these merging points represents zones of influence (Fig. 35.10b) of each water body. The geometric organizations of these 

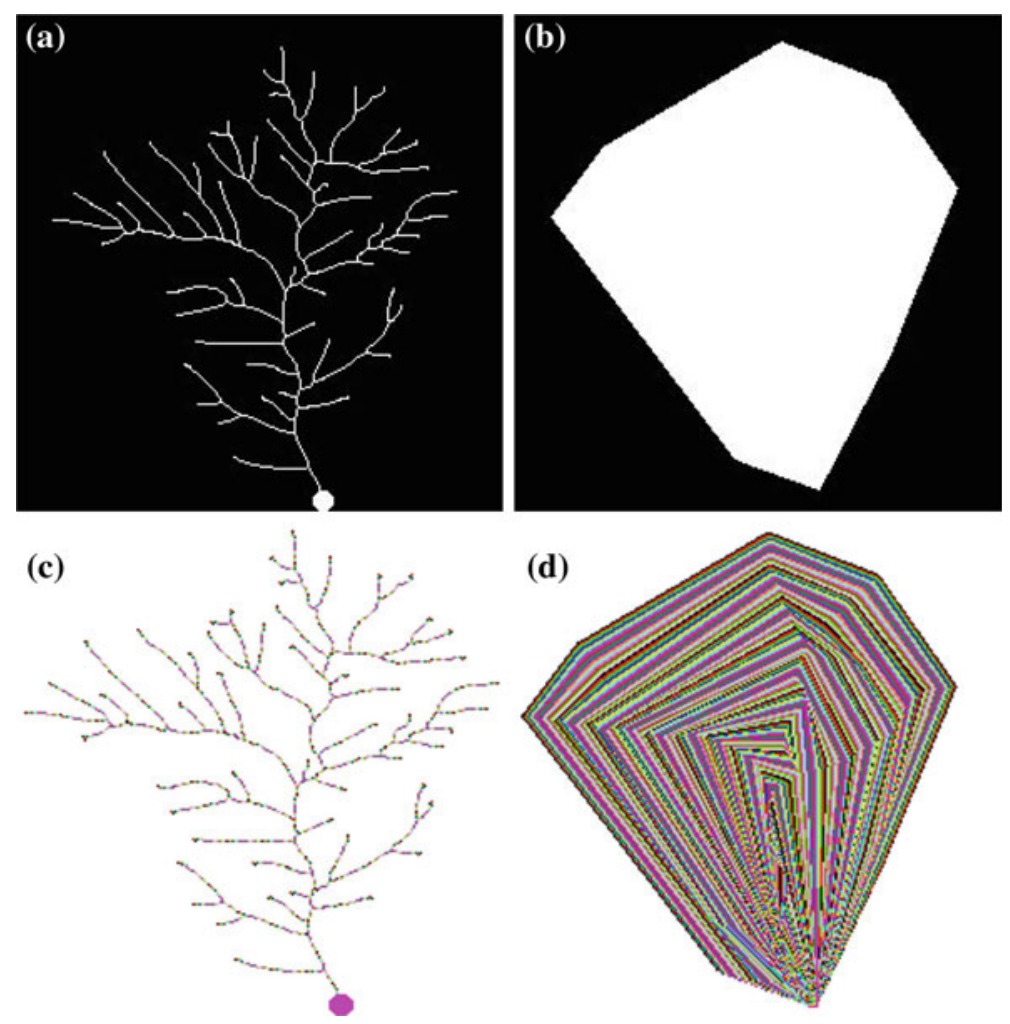

Fig. 35.7 a An example of fourth-order channel network (nonconvex set) and $\mathbf{b}$ its convex hull. A stationary outlet is shown as a round dot in Fig 35.7a. c color-coded traveltime network pruned iteratively until it reaches the outlet and $\mathbf{d}$ color-coded union of convex hulls of networks pruned to different degrees

two phenomena are respectively sensitive and insensitive to perturbation due to exogenic processes. To demonstrate the allometric relationships of water bodies and their zones of influence, a large number of surface water bodies (irrigation tanks), situated in the floodplain region of certain rivers of India, which are retrieved from multi-date remotely sensed data were analyzed in 2-D space (Sagar et al. 1995a, b). Basic measures of these water bodies obtained by morphological analysis were employed to show fractal-length-area-perimeter relationships.

We found that these phenomena follow the universal scaling laws (Sagar et al. 2002; Sagar 2005a, b) found in other geophysical and biological contexts. In this work, universal scaling relationships among basic measures such as area, length, diameter, volume, and information about networks are exhibited by several natural phenomena to further retrieve and understand the common principles underlying organization of these phenomena. Some of the recent findings on universal scaling relations include relationships between brain and body, length and area (or volume), 
(a)

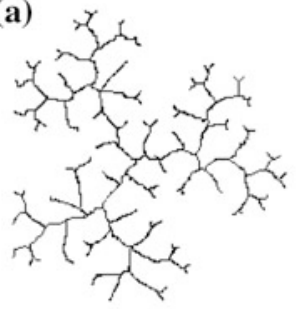

(d)

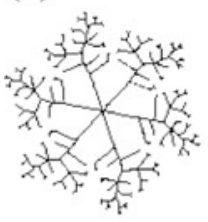

(b)

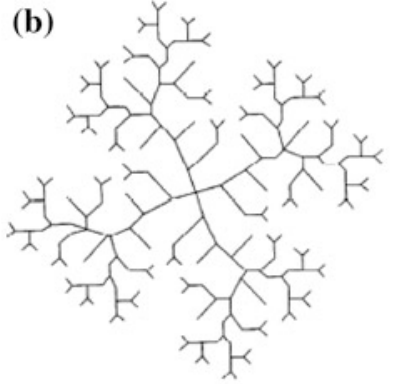

(c)

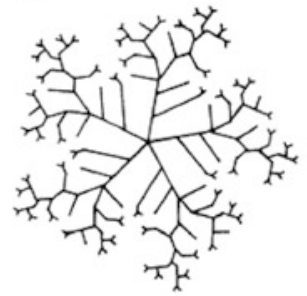

(e)

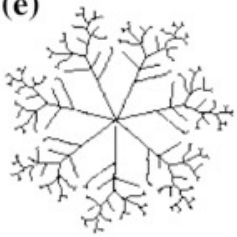

(f)

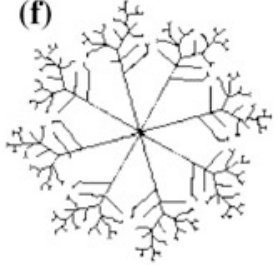

(g)

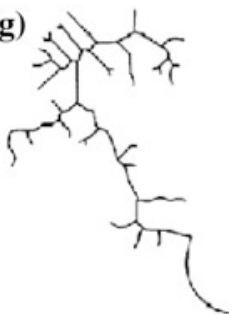

Fig. 35.8 Networks in a three-sided fractal basin, $\mathbf{b}$ four-sided fractal basin, $\mathbf{c}$ five-sided fractal basin, $\mathbf{d}$ six-sided fractal basin, e seven-sided fractal basin, $\mathbf{f}$ eight-sided fractal basin, and g Nizamsagar reservoir. (From Sagar et al. 1998a, b, 1999, 2001)

(a)
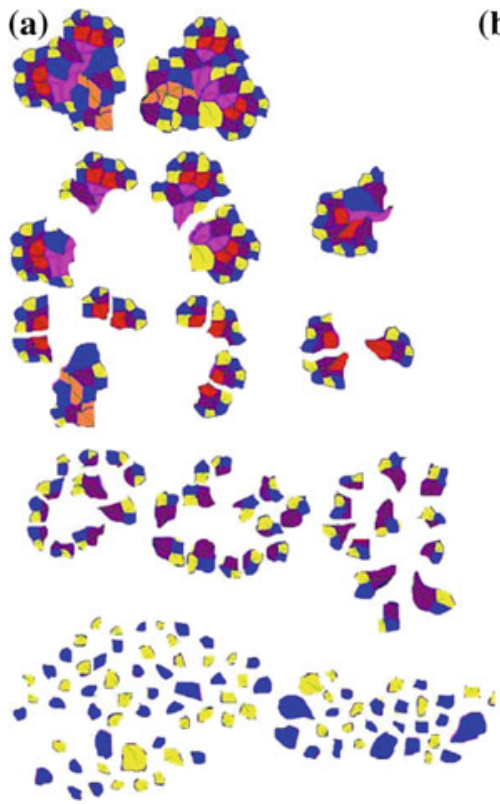

(b)
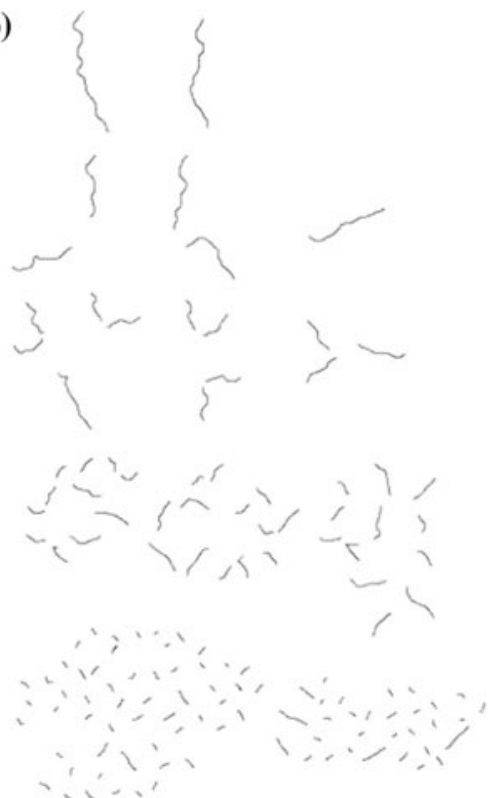

Fig. 35.9 a sub-basins decomposed from a Hortonian F-DEM areas, and $\mathbf{b}$ corresponding main lengths 
(a)

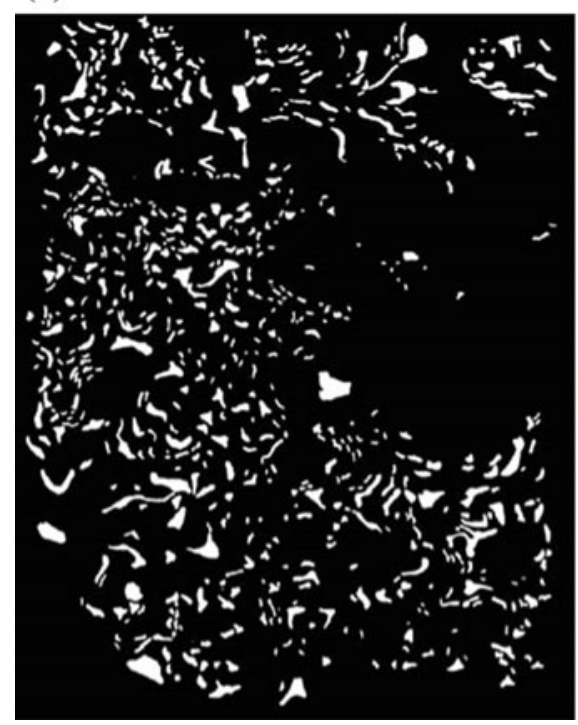

(b)

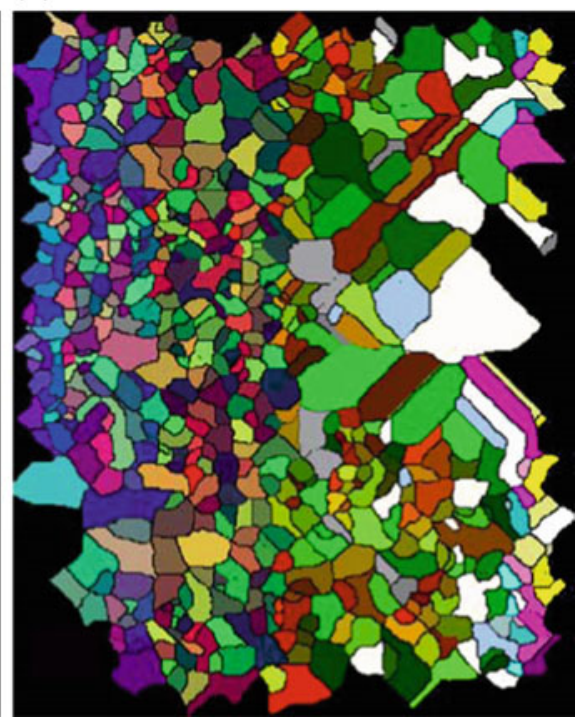

Fig. 35.10 a A section consisting of a large number of small water bodies traced from the floodplain region of Gosthani River and $\mathbf{b}$ zones of influence of water bodies shown in Fig. 35.10a. Different colors are used to distinguish the adjacent influence zones

size and number, size and metabolic rate. In this study, we have shown a host of universal scaling laws in surface water bodies (Fig. 35.10a) and their zones of influence (Fig. 35.10b) that have similarities with several of these relationships encountered in various fields are shown.

\subsubsection{Morphometry of Non-network Space: Scale Invariant but Shape-Dependent Dimension}

In sequel works on terrestrial analysis, we argued that the universal scaling laws shown as examples in earlier section possess limited utility in exploring possibilities to relate them with geomorphologic processes. These arguments formed the basis for alternative methods (Radhakrishna et al. 2004; Teo et al. 2004; Sagar and Chockalingam 2004; Chockalingam and Sagar 2005; Tay et al. 2005a, b, 2007). Shape and scale based indexes provided to analyze and classify non-network space (hillslopes) (Sagar and Chockalingam 2004; Chockalingam and Sagar 2005), and terrestrial surfaces (Tay et al. 2005a, b, 2007) received wide attention. These methods that preserve the spatial and morphological variability yield quantitative results that are scale invariant but shape dependent, and are sensitive to terrestrial surface variations. "Fractal dimension of non-network space of a catchment basin", 

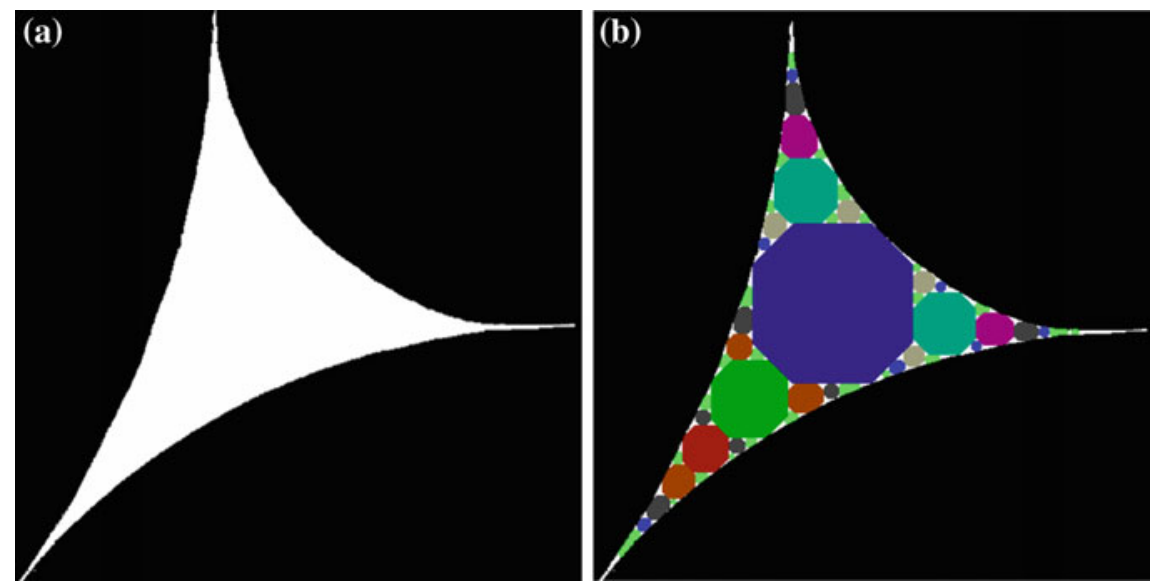

Fig. 35.11 a Apollonian space, and $\mathbf{b}$ after decomposition by means of octagon

provides an approach to show basic distinction between the topologically invariant geomorphologic basins. It introduced morphological technique for hillslope decomposition that yields a scale invariant, but shape dependent, power-laws (Fig. 35.11a, b).

Varied degrees of topographically convex regions within a catchment basin represent varied degrees of hill-slopes. The non-network space, the characterization of which we focused on in our investigations, is akin to the space that is achieved by subtracting channelized portions contributed due to concave regions from the watershed space. This non-network space is akin to non-channelized convex region within a catchment basin. We proposed an alternative shape-dependent quantity akin to fractal dimension to characterize this non-network space (e.g.: Fig. 35.12a). Towards this goal, non-network space is decomposed, in two- dimensional discrete space, into simple non-overlapping disks (NODs) of various sizes by employing mathematical morphological transformations and certain logical operations (Fig. 35.12b). Furthermore, number of NODs of lesser than threshold radius is plotted against the radius, and computed the shape-dependent fractal dimension of non-network space. This study was extended to derive shape dependent scaling laws as the laws derived from network measurements are shape independent for realistic basins (Fig. 35.12). The relationship between number of NODs and the radius of the disk provides an alternative fractal-like dimension that is shape dependent. This was done with the aim to relate shape dependent power laws with geomorphic processes such as hill-slope processes and erosion.

Applications of mathematical morphology transformations are shown to decompose fractal basins (e.g.: Fig. 35.11a) into non-overlapping disks of various sizes (Fig. 35.11b) further to derive fractal power-laws based on number-radius relationships. 

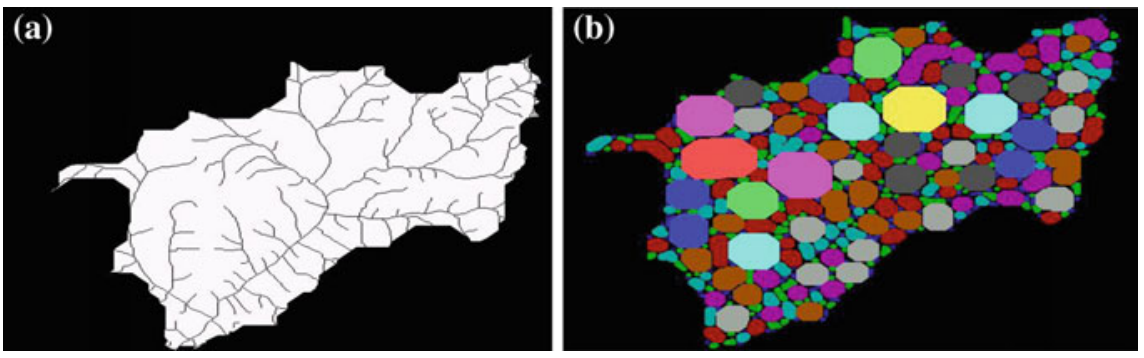

Fig. 35.12 a 5th order channel network $\mathbf{c}$ of Durian Tungal catchment basin, basin $\mathrm{X}$ is reconstructed from this channel network via multiscale morphological closing transformation, b $\mathrm{M}=\mathrm{X} \backslash \mathrm{C}$

\subsubsection{Geodesic Spectrum}

We have provided a novel geomorphologic indicator by simulating geodesic flow fields (Fig. 35.13d-f) within basins (Fig. 35.13a-c) consisting of spatially distributed elevation regions (Lim and Sagar 2008a, b), further to compute a geodesic spectrum that provides a unique one-dimensional geometric support.

This one-dimensional geometric support, in other words geodesic spectrum, outperforms the conventional width-function based approach which is usually derived from planar forms of basin and its networks-construction involves basin as
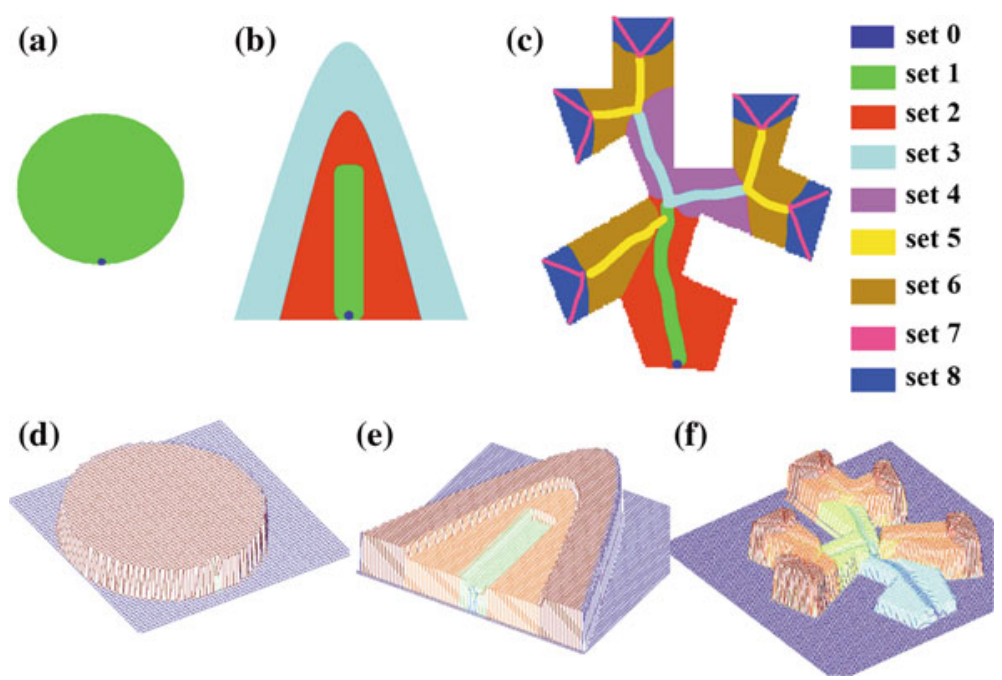

Fig. 35.13 a a flat circular basin, $\mathbf{b}$ a basin with three spatially distributed elevation regions, $\mathbf{c}$ a fractal basin with channelised and non-channeled regions $\mathbf{d}$ flow fields with isotropic propagation in $\mathbf{a}, \mathbf{e}$ isotropic flow fields within $\mathbf{b}$, and $\mathbf{f}$ flow fields within $\mathbf{c}$ and orthogonality between the flow fields of channelized and non-channelized zones is obvious. (From Lim and Sagar 2008a, b.) 

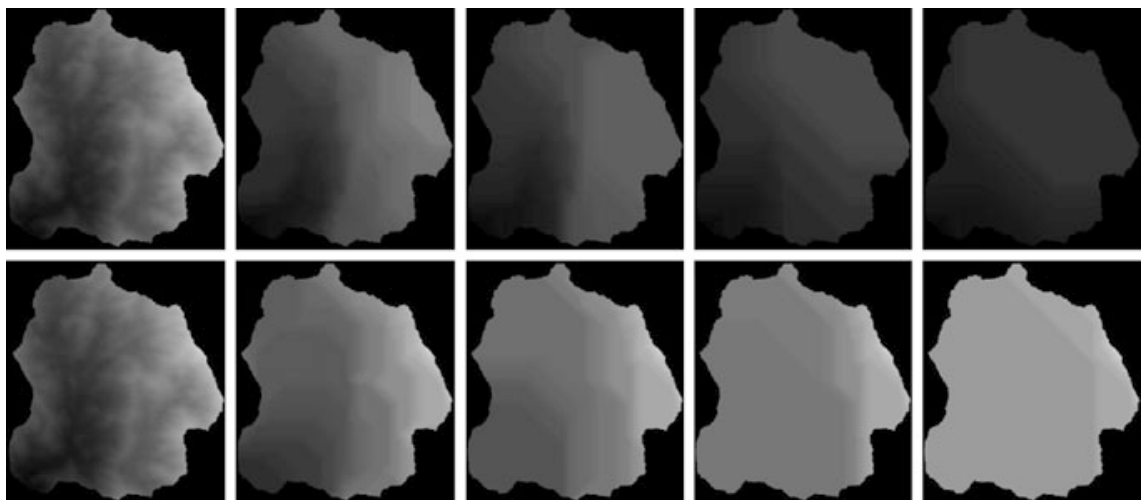

Fig. 35.14 Basin 1 of Cameron Highlands is taken as an example to show the basin images at multiple scales generated via closing and opening. Basin 1 is located at the northern part of Cameron Highlands region, with a size of $3.1 \mathrm{~km}$ (east to west) $63.4 \mathrm{~km}$ (north to south). (Upper sequence) DEM at multiple scales generated via opening, and (Lower Panel) multiscale DEMs generated via closing

a random elevation field (e.g. Digital Elevation Model, DEM) and all threshold elevation regions decomposed from DEM for understanding the shape-function relationship much better than that of width function.

\subsubsection{Granulometric and Anti-granulometric Analysis of Basin-DEMs}

Granulometric indexes derived for spatial elevation fields also yield scale invariant but shape-dependent measures (Tay et al. 2005a, b, c, 2007). DEMs are analyzed by following granulometries via multiscale opening (Fig. 35.14 upper panel), and antigranulometries (Fig. 35.14 lower panel) to derive shape-size complexity measures of foreground and background respectively that provide new indices to understand the terrestrial surfaces further to relate with several geomorphic processes.

\subsection{Geomorphologic Modeling and Simulation}

Simulations allow us to gain a significantly good understanding of complex geomorphologic systems in a way that is not possible with lab experiments. Effectively attaining these goals presents many computational challenges, which include the development of frameworks. The robustness of mathematical morphological operators combined with concepts of fractal geometry (Mandelbrot 1982) in 
modeling and simulations of certain geoscientific phenomena and processes is shown briefly with illustrative examples in this section. The phenomena and processes given emphasis in this section include geomorphologic features, basins and channel networks, landscapes, water bodies, symmetrical folds and ideal sand dunes. Besides providing approaches to simulate fractal-skeletal based channel network model and fractal landscapes, we have shown via the discrete simulations the varied dynamical behavioral phases of certain geoscientific processes (e.g. water bodies, ductile symmetric folds, sand dunes, landscapes) under nonlinear perturbations due to endogenic and exogenic nature of forces. For these simulations we employed nonlinear first order difference equations, bifurcation theory, fractal geometry, and nonlinear morphological transformations as the bases. The three complex systems that we focus on include the channelization process, surface water bodies, and elevation structures.

\subsubsection{Geomorphologic Modeling: Concept of Discrete Force}

Concept of discrete force was proposed from theoretical standpoint to model certain geomorphic phenomena, where geomorphologically realistic expansion and contractions, and cascades of these two transformations were proposed, and five laws of geomorphologic structures are proposed (Sagar et al. 1998a, b). A possibility to derive a discrete rule from a geomorphic feature (e.g. lake) undergoing morphological changes that can be retrieved from temporal satellite data was also proposed in this work, and explained (Fig. 35.15). Laws of geomorphic structures under the perturbations are provided and shown, through interplay between numerical simulations and graphic analysis as to how systems traverse through various behavioral phases.
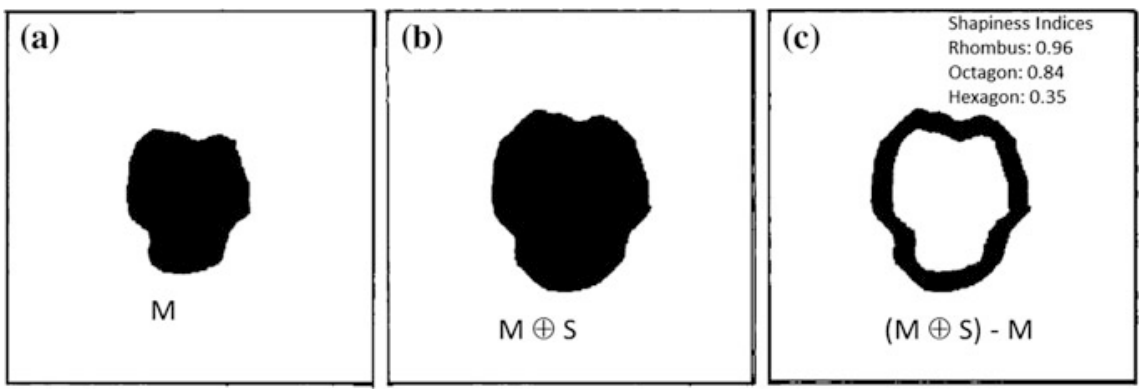

Fig. 35.15 a Hypothetical geomorphic feature at time t, b geomorphic feature at time $t+1$, and c difference in geomorphic feature from time $t$ to $t+1$ 


\subsubsection{Fractal-Skeletal Based Channel Network Model}

Our work on channel network modelling Gastner and Newman (2004) and Sagar (2001c) represents unique contributions to the literature, which until recently were dominated by the classic random model. Fractal-skeletal based channel network model (F-SCN) was proposed by following certain postulates. We developed the Fractal-Skeletal Channel Network (F-SCN) model by employing morphological skeletonization to construct other classes of network models, which can exhibit various empirical features that the random model cannot. In the F-SCN model that gives rise to Horton laws, the generating mechanism plays an important role. Homogeneous and heterogeneous channel networks can be constructed by symmetric generator with non-random rules, and symmetric or asymmetric generators with random rules. Subsequently, F-SCNs (Fig. 35.16d-f) in different shapes of fractal basins (Fig. 35.16a-c) are generated and their generalized Hortonian laws (Fig. 35.16g, h) are computed which are found to be in good accord with other established network models such as Optimal Channel Networks (OCNs), and realistic rivers. F-SCN model is extended to generate more realistic dendritic branched networks.

\subsubsection{Fractal Landscape via Morphological Decomposition}

By applying morphological transformations on fractals of varied types are decomposed into topologically prominent regions (TPRs) (Fig. 35.17a) and each TPR is coded and a fractal landscape organization that is geomorphologically realistic is simulated (Fig. 35.17b) (Sagar and Murthy 2000).

(a)
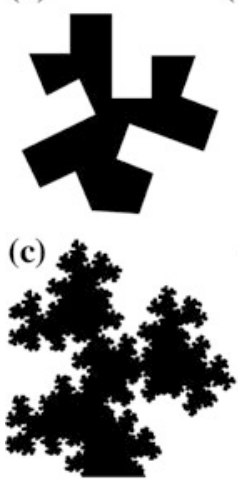

(b)

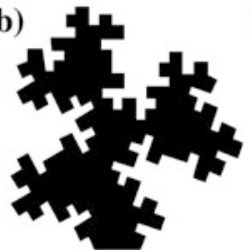

(d)

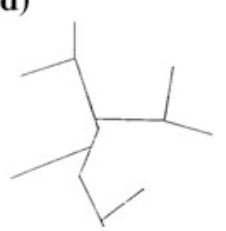

(e)

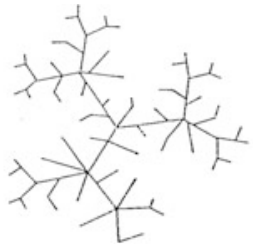

(g)

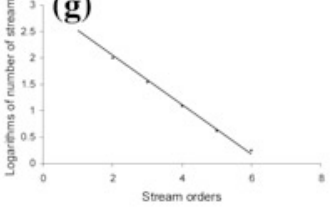

(f)

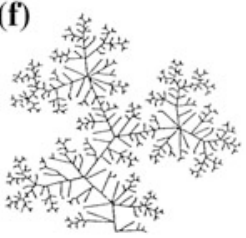

(h)

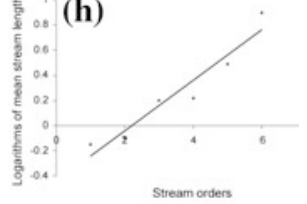

Fig. 35.16 a, b and c Fractal basins after respective iterations. d, e and f An evolutionary sequence of F-SCNs after respective iterations, $\mathbf{g}$ Horton's law of number, and $\mathbf{h}$ Horton's law of mean length 

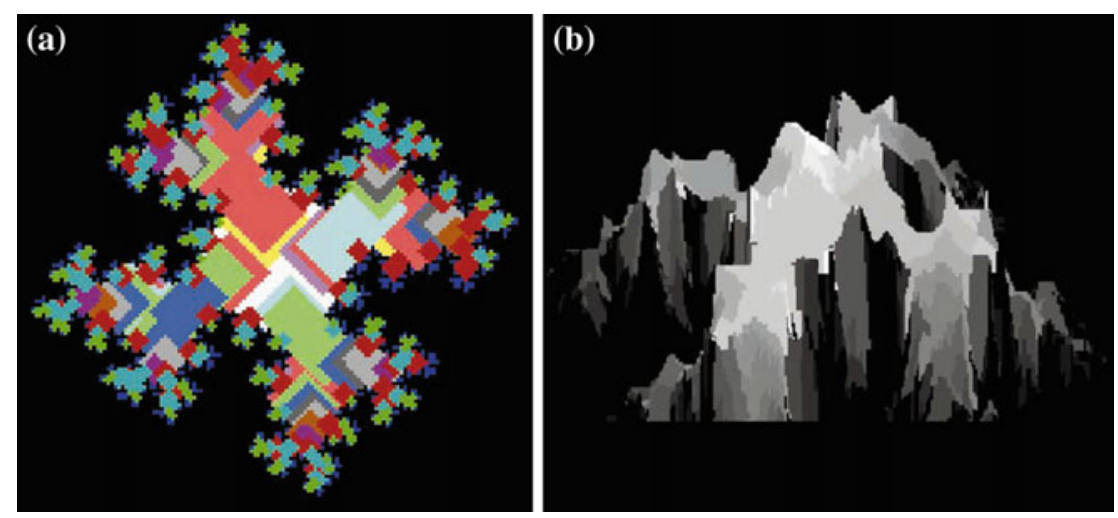

Fig. 35.17 a A binary fractal basin after decomposition into TPRs b A fractal landscape generated from Fig. 35.17a. Light and dark regions of DEM are visualized as high and low elevations (vertical exaggeration: 7)

\subsubsection{Discrete Simulations and Modeling the Dynamics of Small Water Bodies, Symmetrical Folds, and Sand Dunes}

In this subsection we show the fusion of computer simulations and modeling techniques in order to better understand certain terrestrial phenomena and processes with the ultimate goal of developing cogent models in discrete space further to gain a significantly good understanding of complex terrestrial systems in a way that is not possible with lab experiments. The three synthetic phenomena that are explained by generating attractors considered include water bodies (Sagar and Rao 1995a, b, c), symmetrical folds (Sagar 1998), and sand dunes (Sagar 1999b, 2000a, b, 2001a, 2005a, b; Sagar and Venu 2001; Sagar et al. 2003a, b).

\subsubsection{Discrete Simulations and Modeling the Dynamics of Small Water Bodies}

Spatio-temporal patterns of small water bodies (SWBs) under the influence of temporally varied streamflow discharge behaviors are simulated in discrete space by employing geomorphologically realistic expansion and contraction transformations (Fig. 35.18). Expansions and contractions of SWBs to various degrees (e.g. Fig. 35.18B g-1), which are obvious due to fluctuations in streamflow discharge pattern (Fig. 35.18A, a-f), simulate the effects respectively owing to streamflow discharge that is greater or less than mean streamflow discharge. The cascades of expansion-contraction are systematically performed by synchronizing the streamflow discharge (Fig. 35.18A, a-f), which is represented as a template with definite 

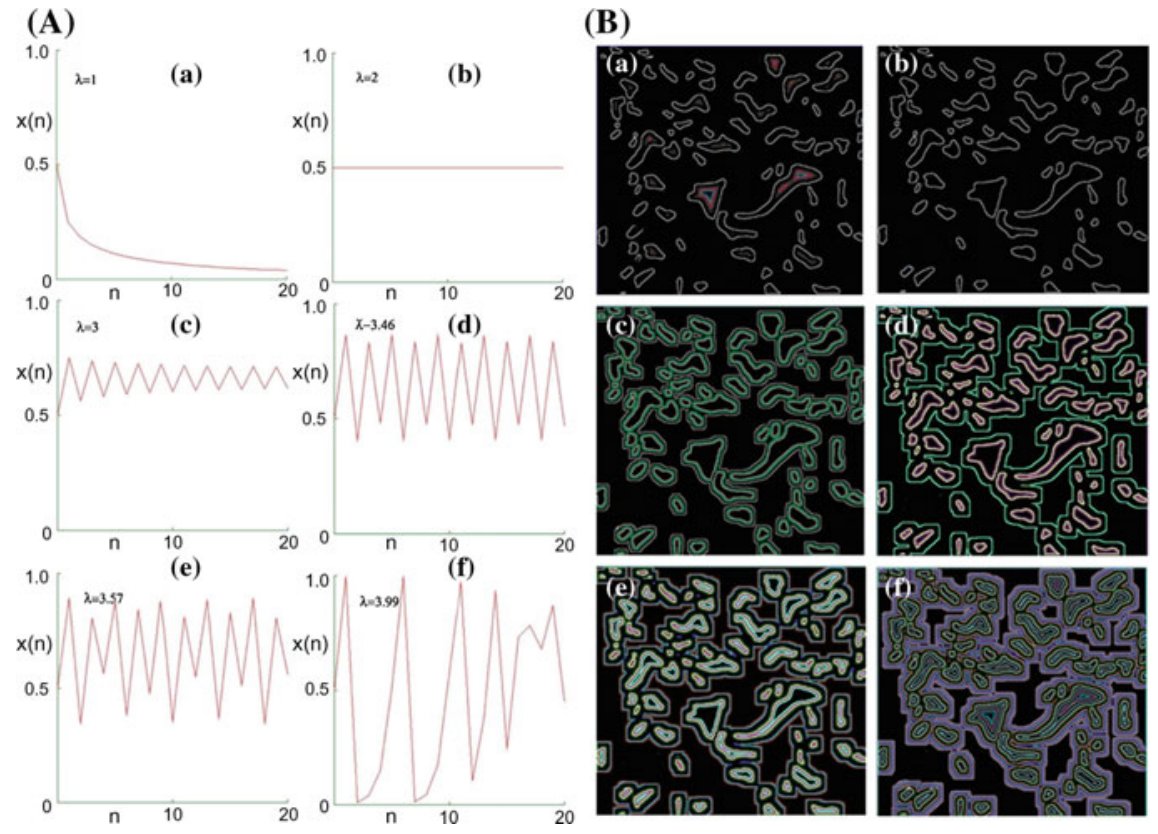

Fig. 35.18 A Streamflow discharge behavioral pattern at different environmental parameters. a-f $\lambda=1,2,3,3.46,3.57$ and 3.99, and $\mathbf{B}$ Spatio-temporal organization of the surface water bodies under the influence of various streamflow discharge behavioral patterns at the environmental parameters at $\mathbf{a}-\mathbf{f} \lambda=1,2,3,3.46,3.57$, and 3.99 are shown up to 20 time steps. In all the cases, the considered initial MSD, A0 $=0.5$ (in normalized scale) is considered under the assumption that the water bodies attain their full capacity. It is illustrated only for the overlaid outlines of water bodies at respective time-steps with various $\lambda \mathrm{s}$

characteristic information, as the basis to model the spatio-temporal organization of randomly situated surface water bodies of various sizes and shapes.

We have shown the varied dynamical behavioral phases of certain geoscientific processes (e.g. water bodies) under nonlinear perturbations via the discrete simulations.

\subsubsection{Ductile Symmetrical Fold Dynamics}

Under various possible time-dependent and time-independent strength of control parameter, in other words nonlinear perturbations, the three-limb symmetrical folds are transformed in a time sequential mode to simulate various possible fold dynamical behaviors (Fig. 35.19a, b) synchronizing trajectory behavior simulated via logistic equation with strength nonlinearity parameters 3.9 and 2.8 (Fig. 35.20a, b). We employed normalized fractal dimension values, and interlimb angles (IAs) as parameters along with strength of nonlinear parameters in this study. Bifurcation 
(a)
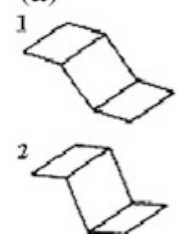

3

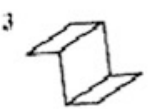

4

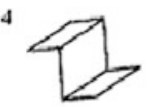

5

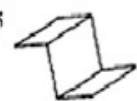

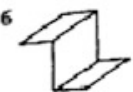
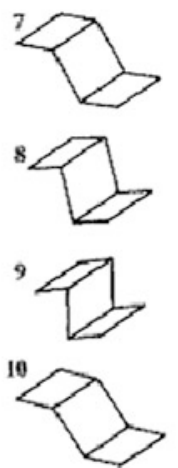
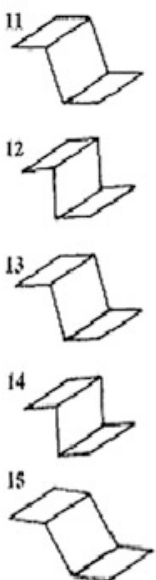

(b)
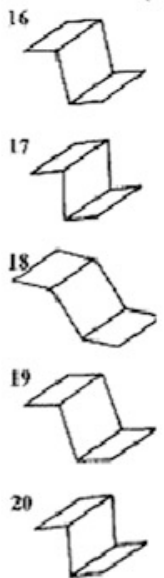
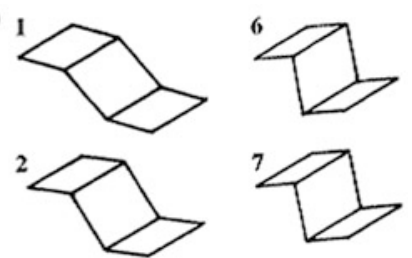

3
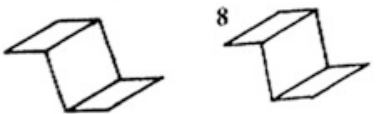

4
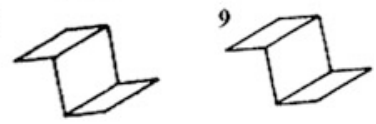

5
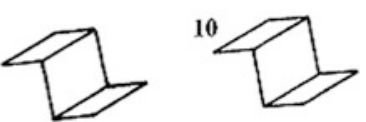

Fig. 35.19 Evolution of a fold type with the strength of nonlinearities: $\mathbf{a} \lambda=3.9$ and $\mathbf{b} \lambda=2.8$. The numbers represent the discrete times. (From Sagar 1998)

diagrams are constructed for both time-dependent and time-independent fold dynamical behaviors, and the equations to compute metric universality by considering the interlimb angles computed at threshold strengths of nonlinearity parameters are proposed (Sagar 1998).

\subsubsection{Symmetrical Sand Dune Dynamics}

Certain possible morphological behaviors with respective critical states represented by inter-slip face angles of a sand dune under the influence of non systematic processes are qualitatively illustrated by considering the first order difference equation that has the physical relevance to model the morphological dynamics of the sand dune evolution as the basis. It is deduced that the critical state of a sand dune under dynamics depends on the regulatory parameter that encompasses exodyanmic processes of random nature and the morphological configuration of sand dune. With the aid of the regulatory parameter, and the specifications of initial state of sand dune, morphological history of the sand dune evolution can be investigated. As an attempt to furnish the interplay between numerical experiments and theory of morphological evolution, the process of dynamical changes (Fig. 35.21) in the sand dune with a change in threshold regulatory parameter (e.g. Fig. 35.22) is modeled qualitatively for a better understanding. An equation to compute metric universality by considering attracting interslipface angles is also proposed. Avalanche size distribution in such a numerically simulated sand dune dynamics have also been studied. 
(a)

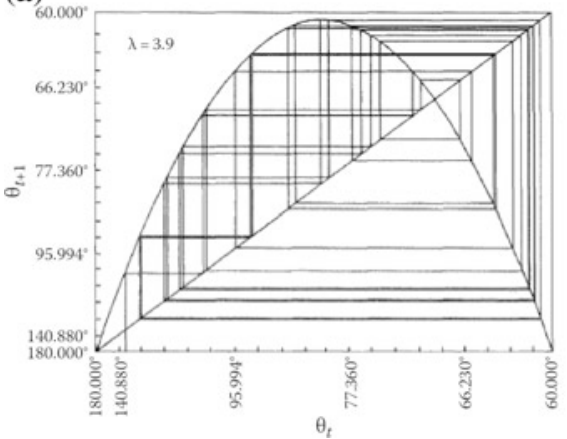

(b)

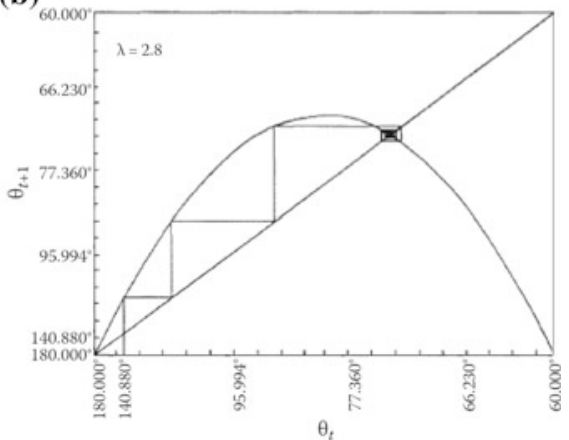

Fig. 35.20 Logistic maps for the qualitative dynamical behavior of symmetric folds under evolution shown in Fig. 35.19a, b. It may be seen that the values mentioned on the abscissa are IAs in degrees for the symmetric fold with three limbs. (From Sagar 1998)

\section{(a) Initial sand dune profile}

(b) $\lambda=3.0$

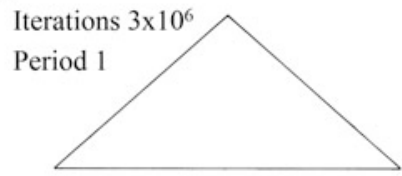

(c) $\lambda=3.46$

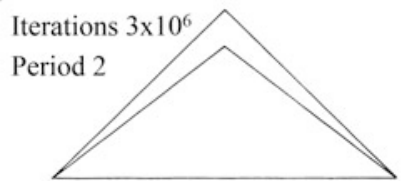

(d) $\lambda=3.569$

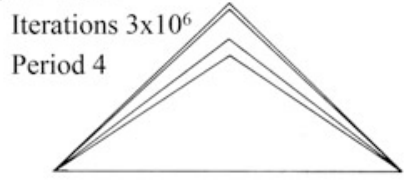

(e) $\lambda=3.57$

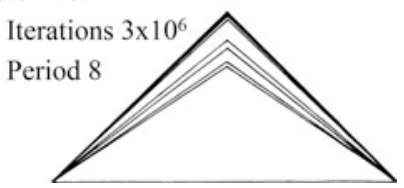

Fig. 35.21 a Initial sand dune profile with $\alpha=0.00001$ or $\theta=179.57334$. The attractor sand dune profiles at various threshold regulatory parameters: $\mathbf{b} \lambda=3$, fixed point attractor sand dune; c $\lambda=3.46$, period 2 attractor sand dunes; $\mathbf{d} \lambda=3.569$, period 4 attractor sand dunes; and $\mathbf{e} \lambda=3.57$, period 8 attractor sand dunes. The attractor sand dune profiles shown in $\mathbf{b}-\mathbf{e}$ are by iterating $3 \times 104$ time steps. (From Sagar 1999a, b)

\subsection{Geospatial Computing and Visualization}

Mathematical morphology not only provides robust solutions in terrestrial pattern retrieval, analysis, and modeling and simulations but also provides numerous insights worth exploring to find solutions for the challenges encountered in GISci. In recent works - that include (i) binary and grayscale morphological interpolations, 


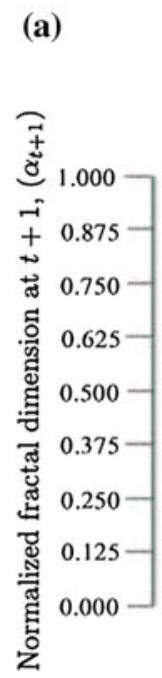
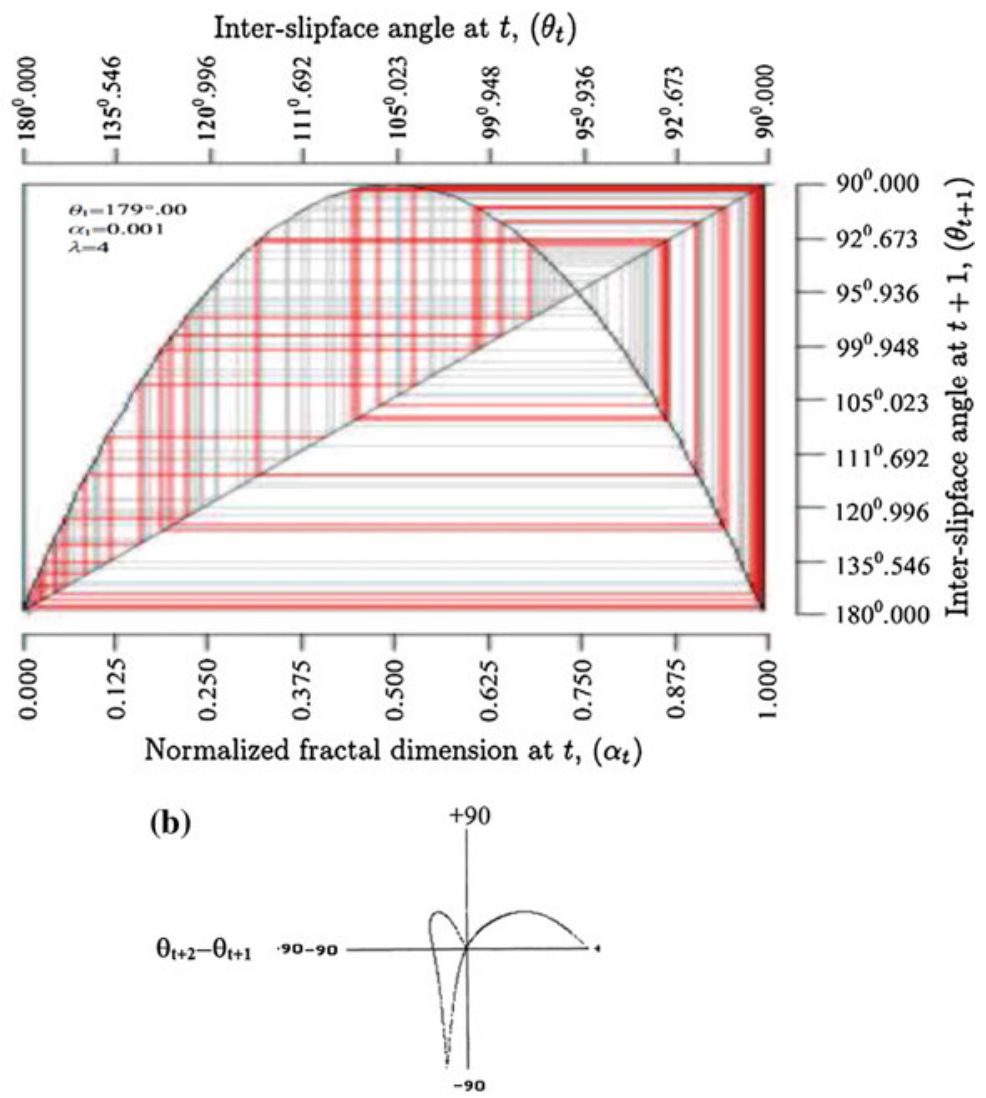

$\theta_{\mathrm{t}+1}-\theta_{\mathrm{t}}$

Fig. 35.22 a A 1-D map plotted between $\theta_{t+1}$ versus $\theta_{t}$ for sand dune case $\lambda=4$ and $\mathbf{b}$ return map plotted between $\theta_{t+1}-\theta_{t}$ versus $\theta_{t+2}-\theta_{t+1}$ for sand dune case with $\lambda=4$. (From Sagar et al. 2003a, b; Sagar and Venu 2001)

SKIZ, WSKIZ and applications in spatiaotemporal visualizations, conversion of point-specific variable data into contiguous zonal maps (Rajashekara et al. 2012), morphing (Sagar and Lim 2015a, b) and variable-specific cartogram generation (Sagar 2014a, b), (ii) volumetric visualization of topologically significant components such as pore-bodies, pore-throats, and pore-channels (Teo and Sagar 2005, 2006), and (iii) spatial reasoning, planning, and interactions (Sagar et al. 2013; Vardhan et al. 2013; Sagar 2018)—one can realize on how robust approaches could be developed by considering mathematical morphological transformations. 


\subsubsection{Morphological Interpolations}

This subsection provides the applications of binary and grayscale morphological interpolations in hierarchical computation of morphological medians and in morphing, and the applications of SKIZ and WSKIZ in conversion of point-specific variable data into contiguous zonal map, and generation of variable-specific contiguous cartograms.

\subsubsection{Computation of Hierarchical Morphological Medians}

Hausdorff-distance based (i) spatial relationships between the maps possessing bijection for categorization and (ii) nonlinear spatial interpolation in visualization of spatiotemporal behavior are proposed and demonstrated. This work (Sagar 2010, 2014a, b; Challa et al. 2016) concerns the development of frameworks with a goal to understand spatial and/or temporal behaviors of certain evolving and dynamic geomorphic phenomena. In (Sagar 2010), we have shown (i) how Hausdorff-Dilation and Hausdorff-Erosion metrics could be employed to categorize the time-varying spatial phenomena, and (ii) how thematic maps in time-sequential mode (Fig. 35.23a) can be used to visualize the spatiotemporal behaviour of a phenomenon, by recursive generation of median elements (Fig. 35.23b). Spatial interpolation, that was earlier seen as a global transform, is extended in Lim and Sagar (2008) by introducing bijection to deal with even connected components. This aspect solves problems of global nature in spatial-temporal GIS. Spatial Interpolation technique is found useful for spatial-temporal GIS and is demonstrated with validation on epidemic spread maps collected for eleven years between 1896 and 1906 (Fig. 35.23a-k, upper left panel). Morphological medians are computed between the epidemic spread maps staggered at two-year interval (Fig. 35.23a-k, upper right panel). Further morphological medians are computed in a hierarchical manner between every two epidemic spread maps of successive years (Fig. 35.23a, b in the lower panel).

\subsubsection{Grayscale Morphological Interpolation and Morphing}

The computation of morphological medians between the thematic maps (binary images) demonstrated in the earlier subsection could be extended to the spatial fields (functions, e.g.: DEMs). This extended version is termed as grayscale morphological interpolation. We have demonstrated the application of grayscale morphological interpolations, computed hierarchically between the spatial fields (Fig. 35.24), to metamorphose a source-spatial field into a target-spatial field. Grayscale morphological interpolations are computed in a hierarchical manner 

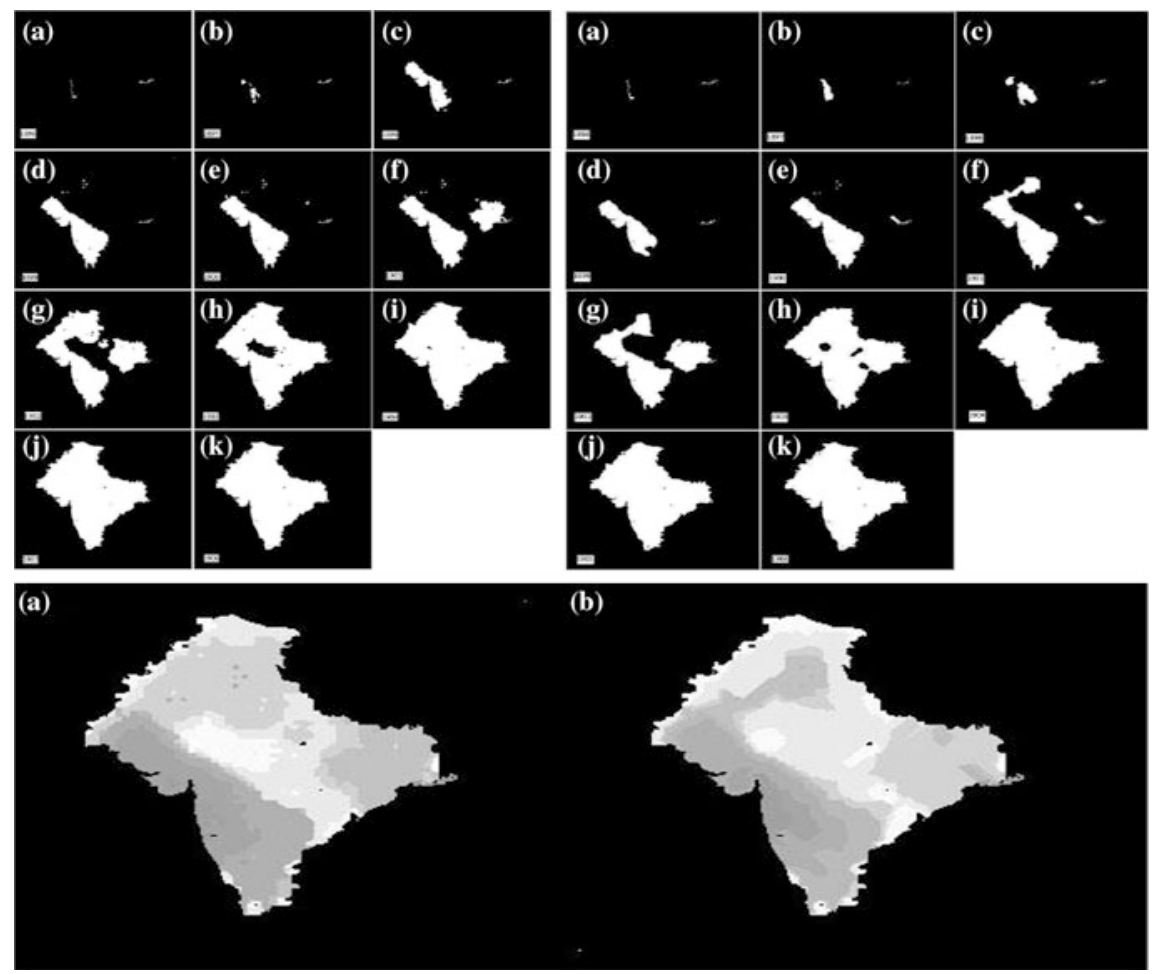

Fig. 35.23 (Upper-Left Panel) a-k Spatial temporal maps that represent the geographic spread of bubonic plague in India between 1896 and 1906 at intervals of one year Maragos and Schafer (1986). The 11 spatial maps depicting the spread of plague were sequentially used to generate the maximum possible number of interpolated maps; (Upper right panel) a Original spatial map of the bubonic plague during 1896. b-j The first level median sets computed for $\mathrm{M}\left(\mathrm{X}^{\mathrm{t}}, \mathrm{X}^{\mathrm{t}+2}\right)$ for all " $t$ " ranging from 1896 to 1905 . k Original spatial map during 1906. For validation, the maps of Fig. $\mathrm{b}-\mathrm{j}$ of upper left panel obtained as first-level median sets are, $\mathrm{M}\left(\mathrm{X}^{\mathrm{t}}, \mathrm{X}^{\mathrm{t}+2}\right)$ respectively, compared for all " $t$ " with those $t$ of Fig. 35.23b-j of upper left panel. These first-level median sets show a reasonable matching with the actual sets (Fig. 35.23b-j of upper left panel); (Lower Panel) Superimposed gray-coded a original spatial maps and $\mathbf{b}$ spatial maps generated via median set computations

(Fig. 35.25) with respect to non-flat structuring element, and found that the morphing, shown for transform source-spatial field into target-spatial field, created with respect to non-flat structuring element is more appropriate as the transition of source-spatial field into the target-spatial field across discrete time steps is smoother than that of the morphing shown with respect to flat structuring element (Sagar and Lim 2015a, b). This morphing shown via nonlinear grayscale morphological interpolations is of immense value in geographical information science, and in particular spatiotemporal geo-visualization. 

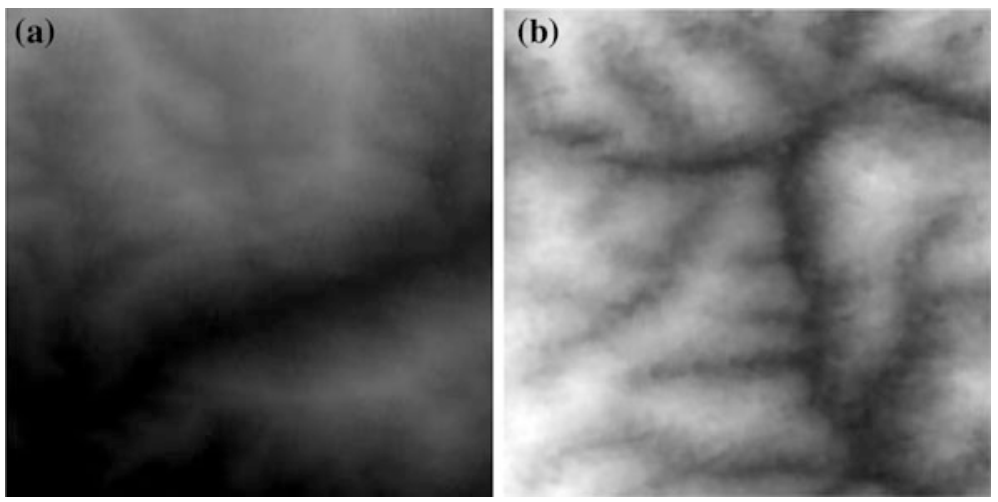

Fig. 35.24 Smaller regions of DEMs: a Cameron Highlands, and $\mathbf{b}$ Petaling region
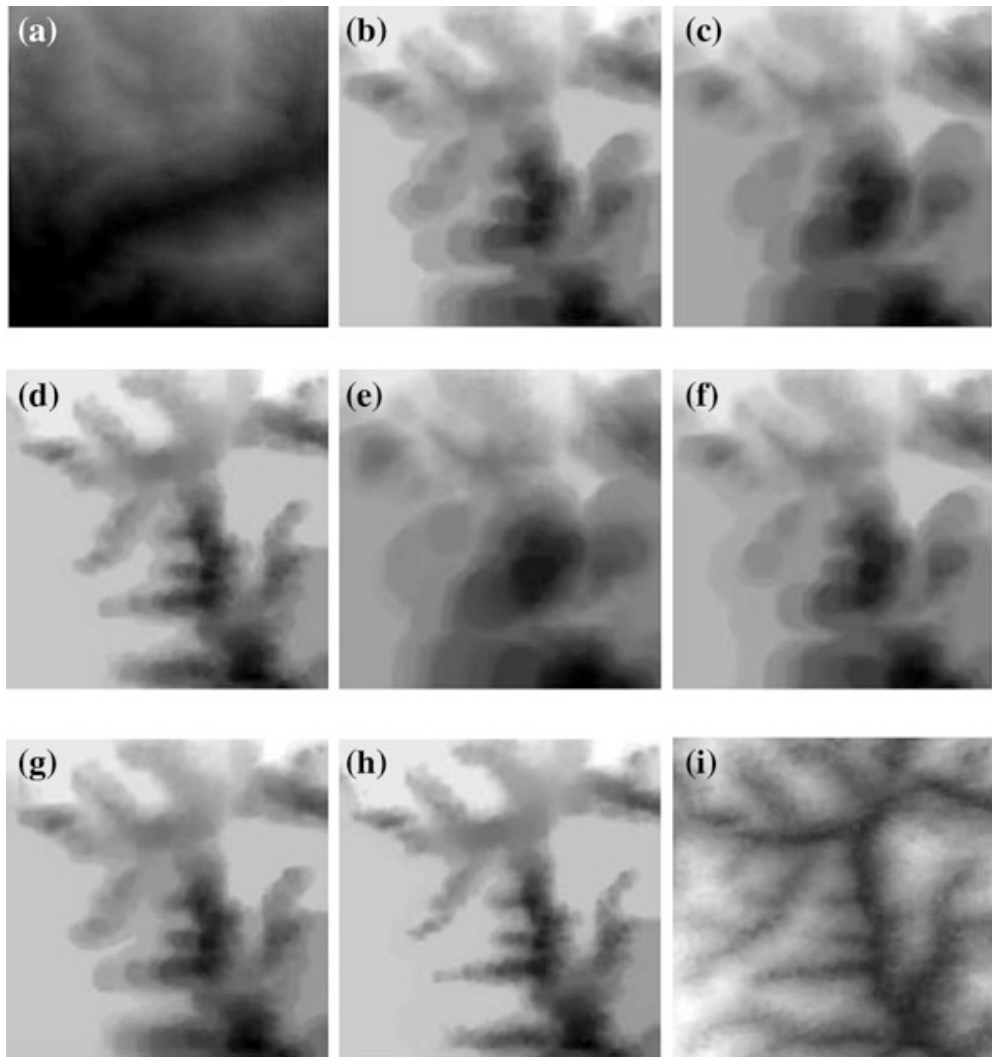

Fig. 35.25 Generation of morphological medians generated by non-flat structuring element, between the DEMs shown in (a) and (i), at b zeroth level, $\mathbf{c}, \mathbf{d}$ first level, and $\mathbf{e}-\mathbf{h}$ second level 


\subsubsection{Point-to-Polygon Conversion via WSKIZ}

Data about many variables are available as numerical values at specific geographical locations in a noncontiguous form. We develop a methodology based on mathematical morphology to convert point-specific data into polygonal data. This methodology relies on weighted skeletonization by zone of influence (WSKIZ). This WSKIZ determines the points of contact of multiple frontlines propagating, from various points (e.g.: gauge stations) spread over the space, at the travelling rates depending upon the variable's strength. We demonstrate this approach for converting rainfall data available at specific rain gauge locations (points) (Fig. 35.26a) into a polygonal map (Fig. 35.26b) that shows spatially distributed zones of equal rainfall in a contiguous form (Rajashekara et al. 2012).

\subsubsection{Cartograms via WSKIZ}

Visualization of geographic variables as spatial objects of size proportional to variable strength is possible via generating variable-specific cartograms. We developed a methodology based on mathematical morphology to generate contiguous cartograms. This approach determines the points of contact of multiple frontlines propagating, from centroids of various planar sets (states), at the travelling rates depending upon the variable's strength (Fig. 35.27a-d).

The contiguous cartogram generated via this algorithm preserves the global shape, and local shapes, and yields minimal area-errors. It is inferred from the comparative error analysis that this approach could be further extended by
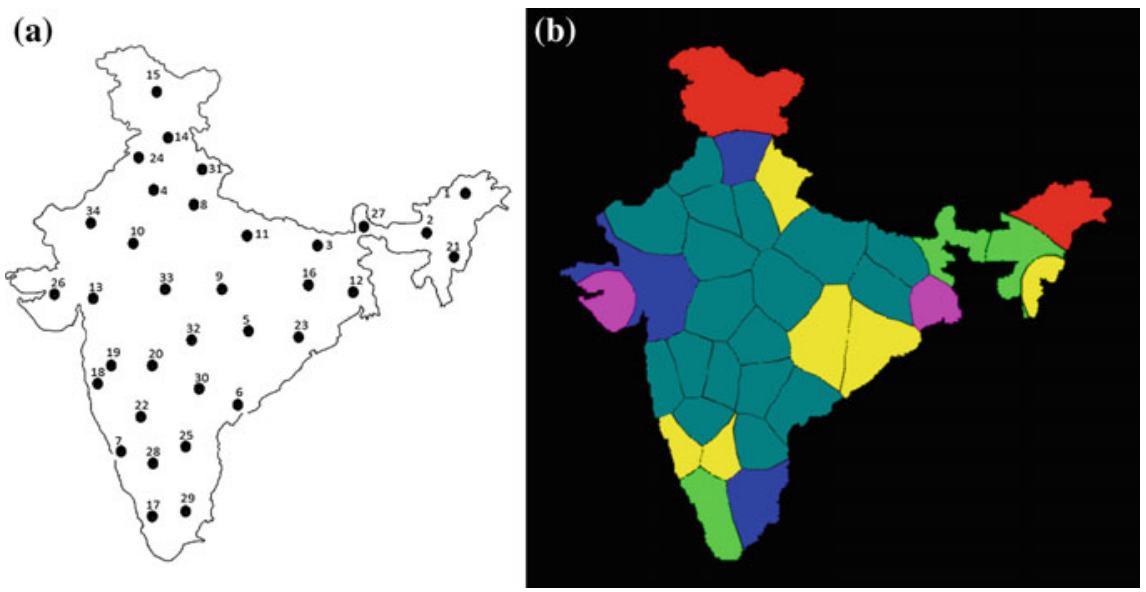

Fig. 35.26 a 34 points (locations) of rain-gauge stations spread over India indexed $\left(A_{1}-A_{34}\right)$, b Rainfall zonal map generated by having various possible propagation speeds, and the variable strengths in terms of propagation speeds 

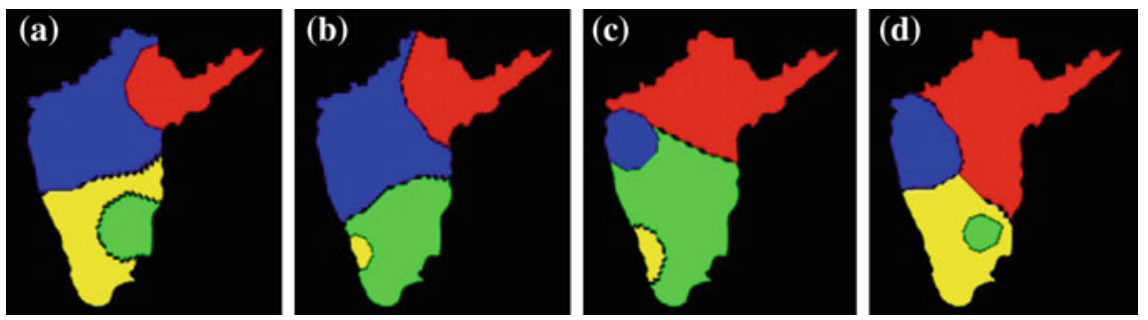

Fig. 35.27 The variable strengths (in terms of propagation speeds are given as $\mathbf{a} A_{2}>A_{4}>A_{1}>A_{3}$, b $A_{2}>A_{1}>A_{3}>A_{4}, \mathbf{c} A_{1}>A_{3}>A_{2}>A_{4}$, and $\mathbf{d} A_{1}>A_{4}>A_{2}>A_{3}$

exploring the applicability of additional characteristics of structuring element, which controls the dilation propagation speed and direction of dilation while generating variable-specific cartograms, to minimize the local shape errors, and area-errors. This algorithm addresses a decade-long problem of preservation of global and local shapes of cartograms. This approach was extended to generate a cartogram for a variable population to demonstrate the proposed approach. Further, the population cartograms for the USA generated via four other approaches (Kocmoud 1997; Keim et al. 2004; Gastner and Newman 2004; Gusein-Zade and Tikunov 1993) are compared with the morphology-based cartogram (Fig. 35.28a-f) in terms of errors with respect to area, local shape, and global shape. This approach for generating cartograms preserves the global shape at the expense of compromising with area-errors. It is inferred from the comparative error analysis that the proposed morphology-based approach could be further extended by exploring the applicability of additional characteristics of probing rule, which controls the dilation propagation speed and direction of dilation while performing WSKIZ, to minimize the local shape errors, and area-errors.

\subsubsection{Visualization of Topological Components in a Volumetric Space}

Heterogeneous material is one that is composed of domains of different materials (phases). The aim of this module is to show how geometric descriptors derived via mathematical morphology and fractal analysis vary between the porous phases isolated from varied types of rocks at various spatial and spectral scales. It is evident from the recent works on Fontainebleau sandstone that the characteristics derived through computer assisted mapping and computer tomographic analysis were well correlated with the physical properties such as porosity, permeability, and conductance. Whatever the physical processes involved in altering the porous phase of material, we propose to emphasise quantifying the complexity of porous phase in both 2-D and 3-D domains. From a petrologic study perspective, such a quantitative characterization in both two- and three-dimensional spaces is of current interest. 
(a)

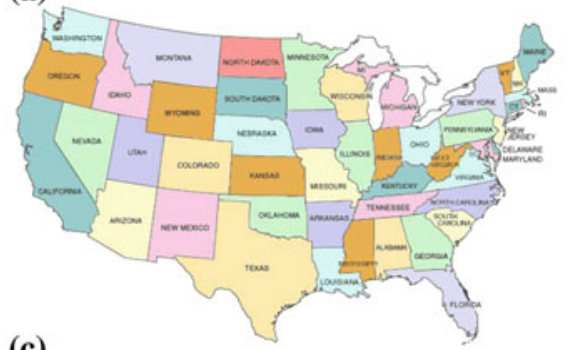

(c)

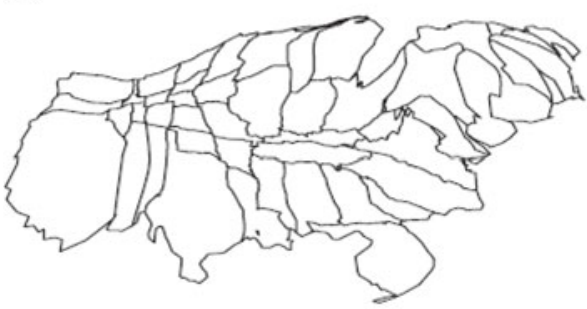

(e)

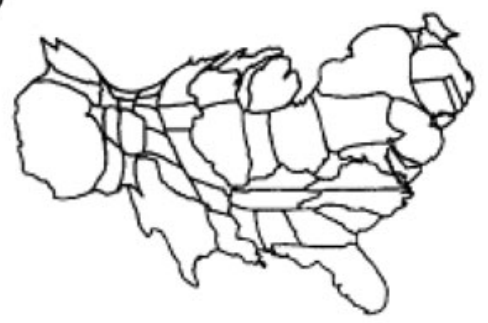

(b)

(d)
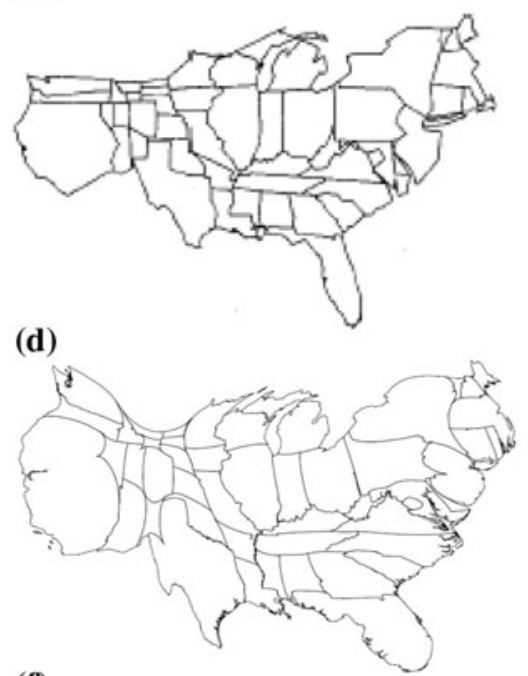

(f)

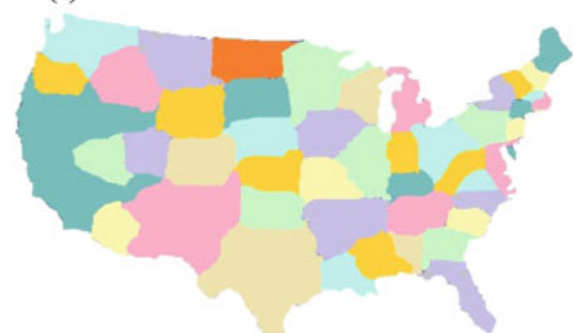

Fig. 35.28 a Equal-area-projection map of USA. b-e Population cartograms generated for USA based on b Continuous cartogram (Kocmoud 1997), c cartodraw (Keim et al. 2004), d Gastner-Newman cartogram (Gastner and Newman 2004), e Area cartogram of the United States, with each county rescaled in proportion to its population (Gusein-Zade and Tikunov 1993), and $\mathbf{f}$ morphology-based cartogram (Sagar 2014a, b). U.S. population cartogram by Gusein-Zade and Tikunov (e: Reproduced with permission from Gusein-Zade and Tikunov 1993, page 172, Fig. 35.1, (C) 1993 American Congress on Surveying and Mapping). The color coding given in Fig. 35.28a is similar to that of Fig. 35.28f

Just like how CT scanning mechanism is employed to scan the brain to study several neurophysiologic processes, one can also employ such a CT-scanning mechanism, besides already existing scanning methods, to scan the rock bodies and store the scanned information in layered forms. Each layer depicts rock's cross sectional information at specific depth. Retrieval of three significant geometric and/ or topologic components, describing organisation of porous medium, that include (a) pore channel, (b) pore throat, and (c) pore body in both 2-D and 3-D spaces is an important task. A 3-D fractal pore (Fig. 35.29a, b) simulated in such a way that it mimics the stacked layers of pore sections is converted into 3-D pore channel 
network (Fig. 35.29c, d), 3-D pore throats (Fig. 35.29e, f) and 3-D pore bodies (Fig. $35.29 \mathrm{~g}, \mathrm{~h}$ ). These decomposed pore features that are of topological importance would shed the light to derive geometric relations which further can be related with that of physical properties of porous structure.
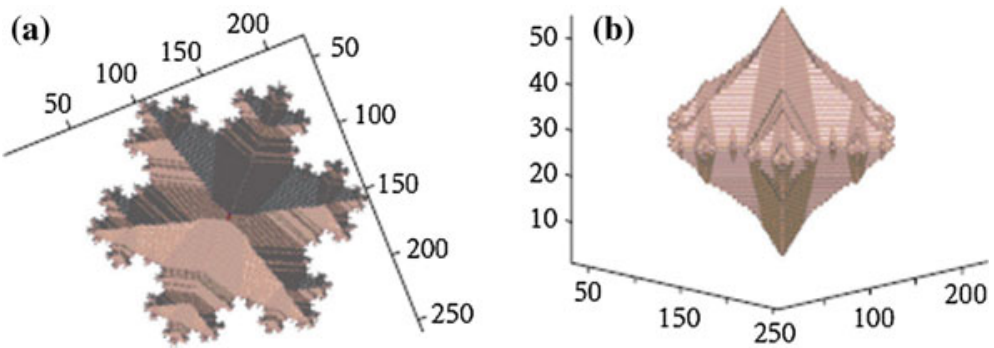

(c)
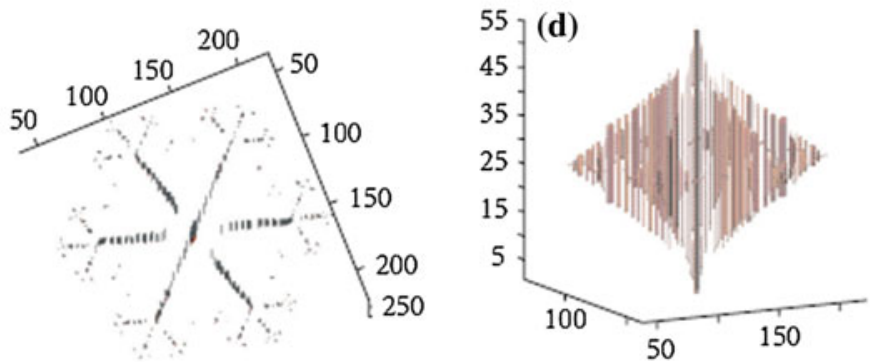

(e)
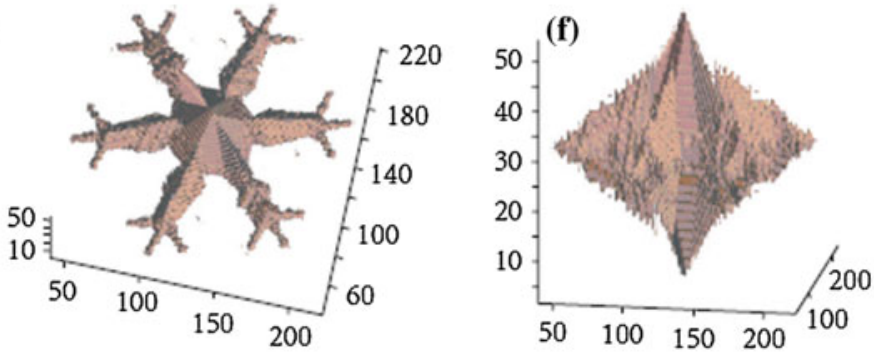

(g)
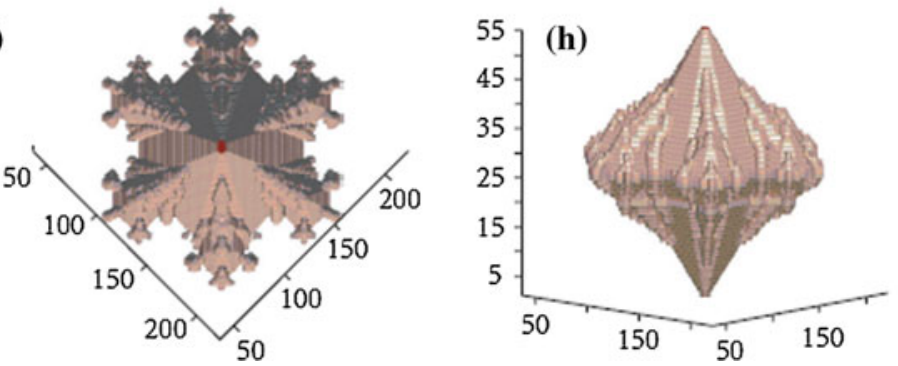

Fig. 35.29 Top and side views of a, b model 3D fractal binary pore, c, d pore-channel, $\mathbf{e}, \mathbf{f}$ pore-throat, and $\mathbf{g}, \mathbf{h}$ pore-body. (Source Teo and Sagar 2006) 


\subsubsection{Spatial Reasoning and Planning}

Mathematical morphology based algorithms developed and demonstrated shown in this subsection include to determine (i) strategically significant set(s) for spatial reasoning and planning, (ii) directional spatial relationship between areal objects (e.g.: lakes, states, sets) via origin-specific dilations, and (iii) spatial interactions via modified gravity model.

\subsubsection{Strategically Significant State $(\mathbf{S})$}

Identification of a strategically significant set from a cluster of adjacent and/or non-adjacent sets depends upon the parameters that include size, shape, degrees of adjacency and contextuality, and distance between the sets. An example of cluster of sets includes continents, countries, states, cities, etc. The spatial relationships, deciphered via the parameters cited above, between such sets possess varied spatial complexities. Hausdorff dilation distance between such sets is considered to derive automatically the strategic set among the cluster of sets. The (i) dilation distances, (ii) length of boundary being shared, and (iii) degrees of contextuality and adjacency between origin-set and destination sets, which together provide solutions to derive strategically significant sets with respect to distance, degree of contextuality, degree of adjacency and length of boundary being shared. Simple mathematical morphologic operators and certain logical operations are employed in this study. Results drawn (Fig. 35.30) - by applying the proposed framework on a case study that involves spatial sets (states) decomposed from a spatial map depicting the country of India—are shown in Fig. 35.30.

This approach has been applied on data depicting randomly spread surface water bodies (Fig. 35.31a, b) and their corresponding zones of influence (Fig. 35.31c, d) within a subbasin to detect the strategically significant water body and zone of influence (Fig. 35.32a, b).

\subsubsection{Directional Spatial Relationship}

We provide an approach to compute origin-specific morphological dilation distances between planar sets (e.g.: areal objects, spatially represented countries, states, cities, lakes) to further determine the directional spatial relationship between sets. Origin chosen for a structuring element that yields shorter dilation distance than that of the other possible origins of structuring element determines the directional spatial relationship between $A_{\mathrm{i}}$ (origin-set) and $A_{\mathrm{j}}$ (destination set). We demonstrate this approach on a cluster of spatial sets (states) decomposed from a spatial map depicting country India (Fig. 35.33a). This approach has potential to extend to any number (type) of sets on Euclidean space. 
(A)

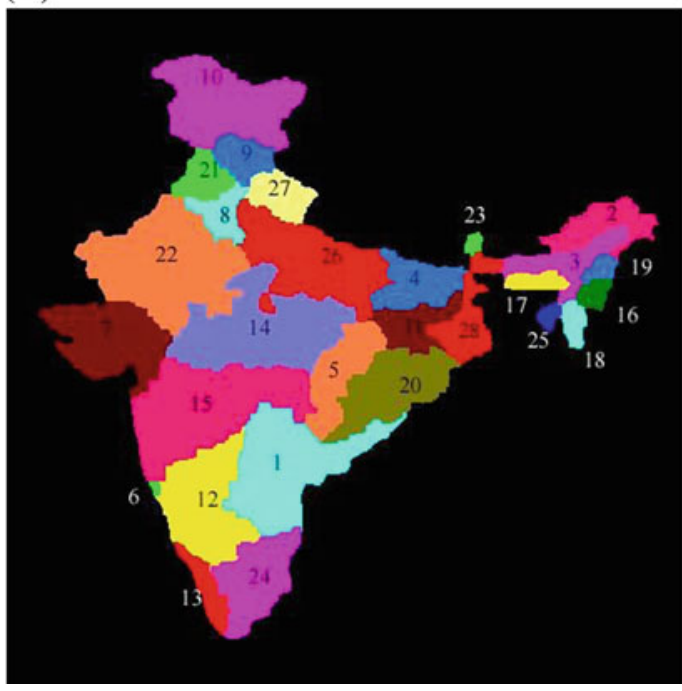

(B)
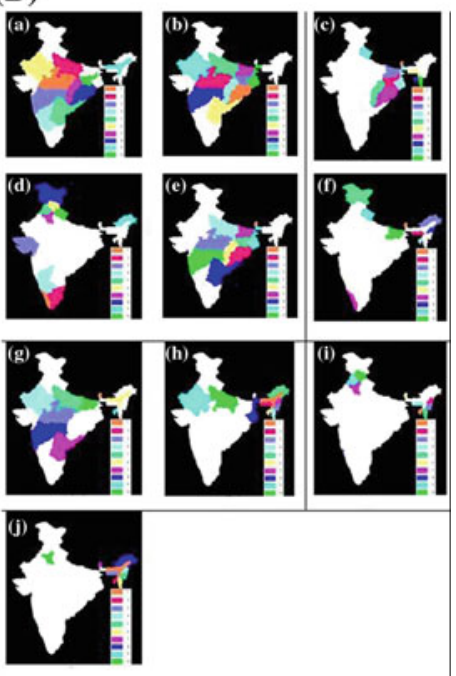

Fig. 35.30 A Map of India (spatial system) with its constituent 28 states (subsets)—indexed according to alphabetical order are shown-Andhra Pradesh $\left(\mathrm{A}_{1}\right)$, Arunachal Pradesh $\left(\mathrm{A}_{2}\right)$, Assam $\left(A_{3}\right)$, Bihar $\left(A_{4}\right)$, Chhattisgarh $\left(A_{5}\right)$, Goa $\left(A_{6}\right)$, Gujarat $\left(A_{7}\right)$, Haryana $\left(A_{8}\right)$, Himachal Pradesh $\left(A_{9}\right)$, Jammu \& Kashmir $\left(A_{10}\right)$, Jharkhand $\left(A_{11}\right)$, Karnataka $\left(A_{12}\right)$, Kerala $\left(A_{13}\right)$, Madhya Pradesh $\left(A_{14}\right)$, Maharashtra $\left(A_{15}\right)$, Manipur $\left(A_{16}\right)$, Meghalaya $\left(A_{17}\right)$, Mizoram $\left(A_{18}\right)$, Nagaland $\left(A_{19}\right)$, Orissa $\left(A_{20}\right)$, Punjab $\left(A_{21}\right)$, Rajasthan $\left(A_{22}\right)$, Sikkim $\left(A_{23}\right)$, Tamilnadu $\left(A_{24}\right)$, Tripura $\left(A_{25}\right)$, Uttarapradesh $\left(A_{26}\right)$, Uttarakhand $\left(A_{27}\right)$, West Bengal $\left(A_{28}\right)$, Union territories and Himalayan hill range that are parts Indian peninsular are not included in the figure. B Spatial representation of strategically important states in the order from 1 to 10 are shown in terms of twelve different parameters shown in Fig. 35.7. In each panel of this Figure, first 10 strategically significant states (please refer to the legend on each panel) are shown in different colors. These strategically significant sets with respect to a boundary being shared, b shortest distance from origin to destination states, $\mathbf{c}$ shortest total distance from destination states to origin state, $\mathbf{d}$ contextuality, e Hausdorff dilation distance, $\mathbf{f}$ spatial complexity involved in length of the boundary being shared, $\mathbf{g}$ spatial complexity in terms of contextuality, $\mathbf{h}$ spatial complexity in terms of distance from origin to destination states, $\mathbf{i}$ spatial complexity in terms of distance from destination states to origin state, $\mathbf{j}$ spatial complexity in terms of Hausdorff dilation distance from origin state to destination states. States with color-codes denote first ten strategically significant states, and the region with white space represents the states that are strategically non-significant with ranks starting from eleven to twenty eight

\subsubsection{Spatial Interactions}

Hierarchical structures include spatial system (e.g. river basin), clusters of a spatial system (e.g. watersheds of a river basin), zones of a cluster (e.g. subwatersheds of a watershed), and so on. Variable-specific classification of the zones of a cluster of zones within a spatial system is the main focus of this work on spatial interactions. Variable-specific (e.g. resources) classification of zones is done by computing the levels of interaction between the $i$ th and $j$ th zones. Based on a heuristic argument, 


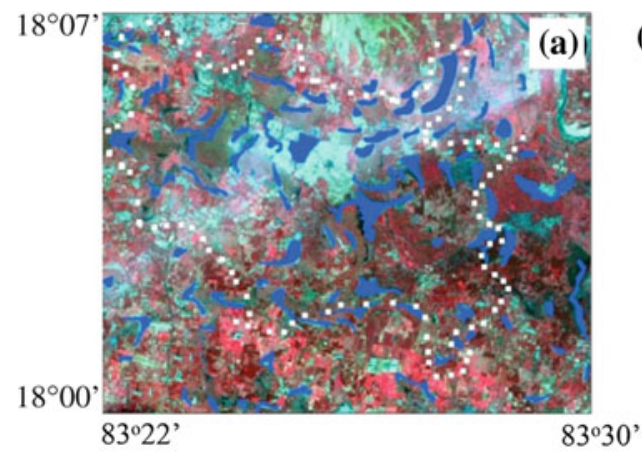

(b)

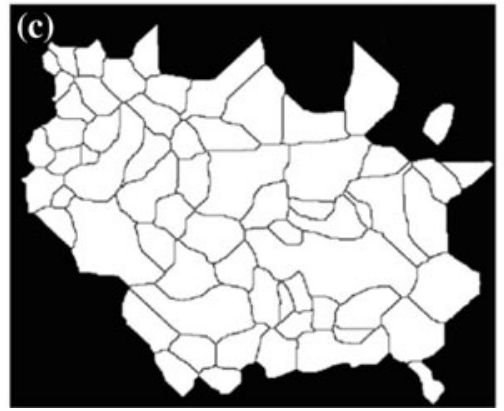

Fig. 35.31 a Indian Remote Sensing satellite (IRS LISS-III) multispectral image of the study area, and the blue objects are water bodies traced from IRS LISS-III image with topographic map reference superposed on IRS LISS-III image, and white dots indicate the boundary of the considered cluster, $\mathbf{b}$ small water bodies, $\mathbf{c}$ zones of influence of corresponding water bodies, and d water bodies and zones of influence with labeling

(a)

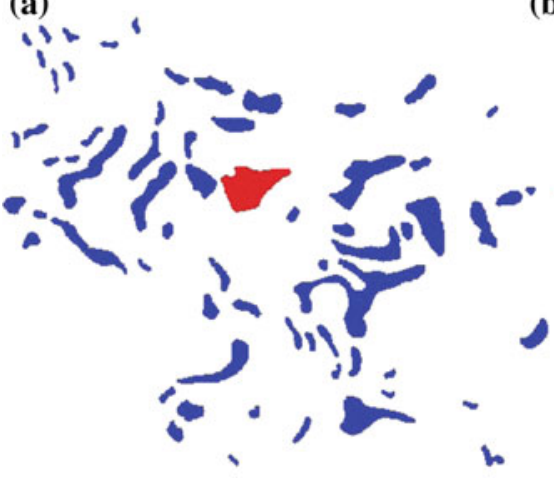

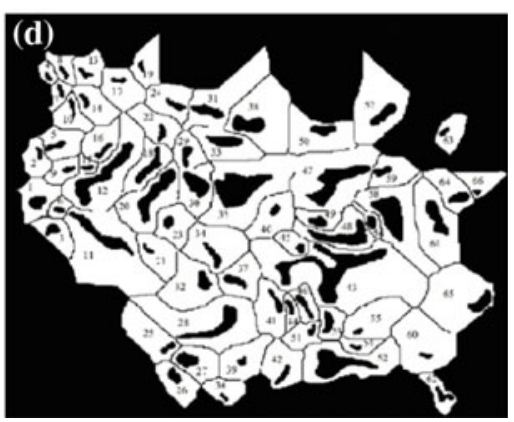

(b)

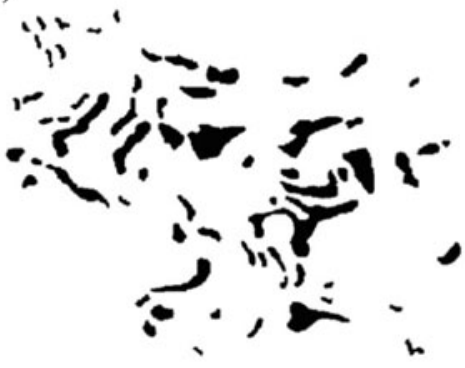


(a)

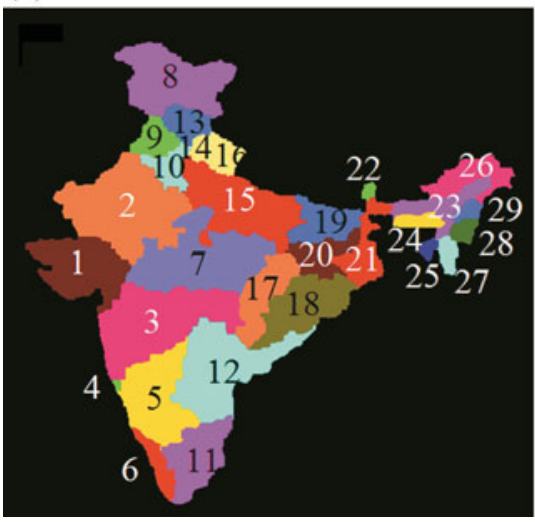

(b)

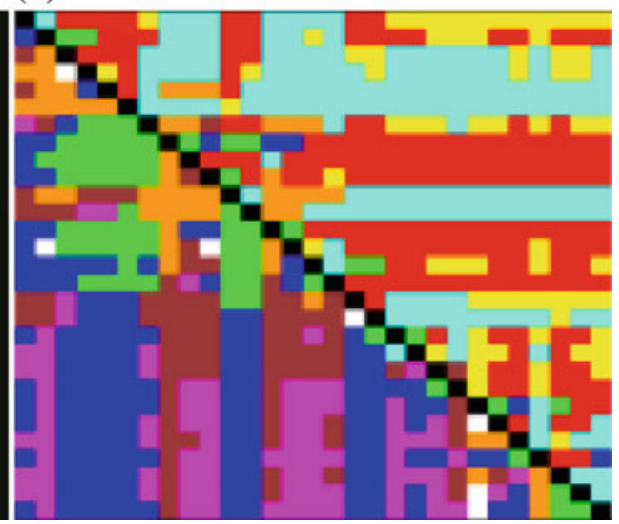

Fig. 35.33 a Twenty nine sets (states of India) indexed according to alphabetical order are shown - Gujarat $\left(A_{1}\right)$, Rajasthan $\left(A_{2}\right)$, Maharashtra $\left(A_{3}\right)$, Goa $\left(A_{4}\right)$, Karnataka $\left(A_{5}\right)$, Kerala $\left(A_{6}\right)$, Madhya Pradesh $\left(A_{7}\right)$, Jammu and Kashmir $\left(A_{8}\right)$, Punjab $\left(A_{9}\right)$, Haryana $\left(A_{10}\right)$, Tamilnadu $\left(A_{11}\right)$, Andhra Pradesh $\left(A_{12}\right)$, Himachal Pradesh $\left(A_{13}\right)$, Delhi $\left(A_{14}\right)$, Uttar Pradesh $\left(A_{15}\right)$, Uttaranchal $\left(A_{16}\right)$, Chhattisgarh $\left(A_{17}\right)$, Orissa $\left(A_{18}\right)$, Bihar $\left(A_{19}\right)$, Jharkhand $\left(A_{20}\right)$, West Bengal $\left(A_{21}\right)$, Sikkim $\left(A_{22}\right)$, Assam $\left(A_{23}\right)$, Meghalaya $\left(A_{24}\right)$, Tripura $\left(A_{25}\right)$, Arunachal Pradesh $\left(A_{26}\right)$, Mizoram $\left(A_{27}\right)$, Manipur $\left(A_{28}\right)$, Nagaland $\left(A_{29}\right)$. Union Territories are not considered. b Directional spatial relationship shown in colored matrix form in which there are 29 rows and 29 columns and a color in each grid cell explaining directional relationship between each state to other 28 states

we proposed a modified gravity model for the computations of levels of interaction between the zones. This argument is based on the following two facts: (i) the level of interaction between the $i$ th and $j$ th zones, with masses $m_{\mathrm{i}}$ and $m_{\mathrm{j}}$ is direction-dependent, and (ii) the level of interactions between the $i$ th and $j$ th zones with corresponding masses, situated at strategically insignificant locations would be much different (lesser) from that of the $i$ th and $j$ th zones with similar masses but situated at strategically highly significant locations. With the support of this argument, we provide a modified gravity model by incorporating the asymmetrical distances, and the product of location significance indexes of the corresponding zones. This modified gravity model yields level of interaction between the two zones that satisfies the realistic characteristic that is level of interaction between the zones is direction-dependent.

Each state of India is designated with ranks in terms of its (i) location significance index, (ii) strengths of interaction of all states with a specific state, (iii) strengths of interaction with other states, and (iv) strength out of (ii) and (iii) (Fig. 35.34a-d). Further by employing a modified gravity model, 28 states $\left(\mathrm{X}_{1}\right.$ to $\mathrm{X}_{28}$ ) of India (Fig. 35.30A) are paired into best interacting to least interacting pairs with respect to areal extents of states as a variable (Fig. 35.35a-j). 


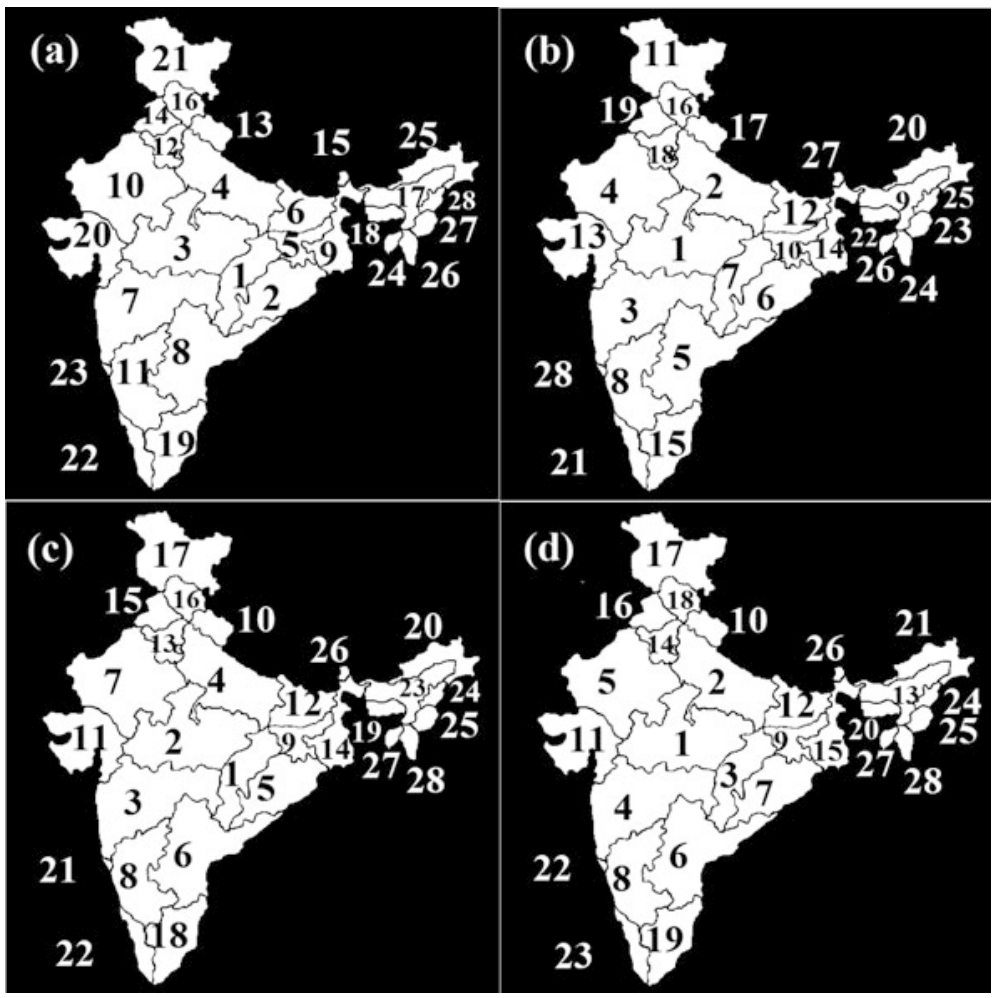

Fig. 35.34 India map with each state designated with a rank with respect to four different parameters. a $\varphi X_{i}, \mathbf{b} \max _{i}\left(\sum_{j} F X_{i j}\right), \mathbf{c} \max _{j}\left(\sum_{i} F X_{j i}\right)$, and $\mathbf{d} \max \left(\max _{i}\left(\sum_{j} F X_{i j}\right), \max _{j}\left(\sum_{i} F X_{j i}\right)\right)$

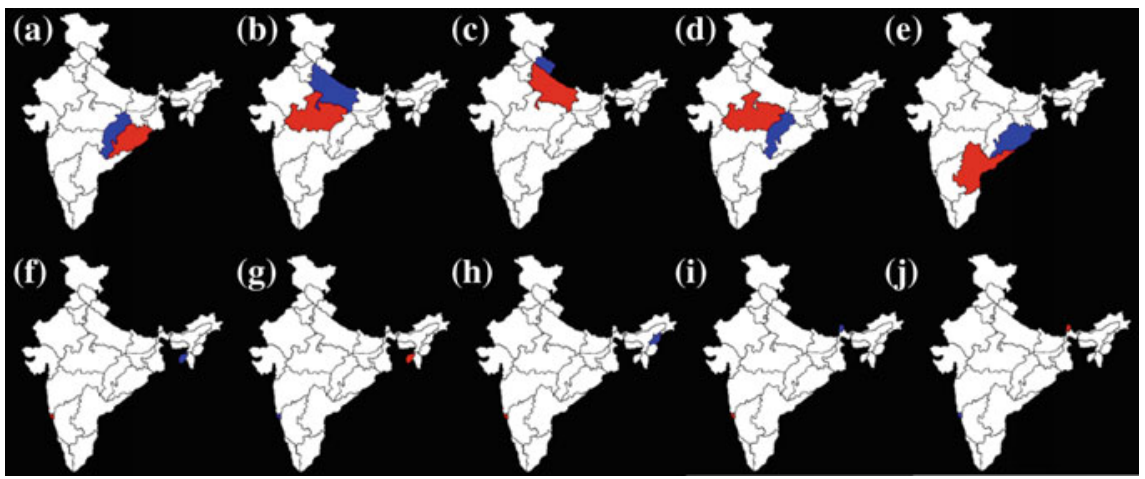

Fig. 35.35 Five best pairs exhibited the high levels of interactions a $X_{20,5}$, b $X_{14,26}$, c $X_{26,27}$, d $X_{14,5}$, and $\mathbf{e} X_{1,20}$. Five pairs exhibited the least levels of interactions $\mathbf{f} X_{6,25}, \mathbf{g} X_{25,6}, \mathbf{h} X_{6,19}$, i $X_{6,23}$, and j $X_{23,6}$ 


\subsection{Conclusions}

From our attempts since early 1990s, we could clearly see a great potential for mathematical morphological transformations in the three aspects (retrieval, analysis and reasoning, and modeling) of relevance to geosciences and GISci. This chapter provided a brief illustrative review on how mathematical morphology could be applied to deal with varied topics of relevance to mathematical geosciences and geographical information science communities. Reader is encouraged to dig cited references for more details. Our studies show that there exist several open problems of relevance to the mathematical geosciences community. These open problems could be well-handled by mathematical morphology. Some of the recent advances of mathematical morphology and their applications in spatial data segmentation and morphological clustering were discussed. Applications of both classical and modern mathematical morphological transformations in geosciences and GISci are yet to be seen in full-length. It is our hope that most visible and highly distinguished scientists who are active in the IAMG activities would spread a word wide across and would spur the interest of young researchers to take the strides forward.

Acknowledgements I would like to gratefully acknowledge Jean Serra and collaborators, mentors, reviewers, examiners, friends, employers, well-wishers, and doctoral students-S.V.L.N. Rao, B.S.P. Rao, M. Venu, K.S.R. Murthy, Gandhi, Srinivas, Radhakrishnan, Lea Tien Tay, Chockalingam, Lim Sin Liang, Teo Lay Lian, Dinesh, Jean Serra, Gabor Korvin, Arthur Cracknell, Deekshatulu, Philippos Pomonis, Peter Atkinson, Hien-Teik Chuah, Laurent Najman, Jean Cousty, Christian Lantuejoul, Alan Tan, VC Koo, Rajesh, Ashok, Pratap, Rajashekhara, Sravan, Aditya, Sampriti, and several others. I am grateful to Frits Agterberg for his phenomenal support and suggestions that made this review chapter readable and understandable.

\section{References}

Agterberg FP (2001) Appreciation of contributions by Georges Matheron and John Tukey to a mineral-resources research project. Nat Resour Res 10(4):287-295

Agterberg FP (2004) Georges matheron: founder of spatial statistics. Earth Sci Hist 23(2):325-334

Beucher S (1990) Segmentation d-images et morphologic mathematique. These Docteur en Morphologic Mathematique. Ecole des Mines de Paris

Beucher S (1999) Mathematical morphology and geology: when image analysis uses the vocabulary of earth science: a review of some applications. In: Proceedings of geovision-99. University of Liege, Belgium, 6-7 May 1999, pp 13-16

Beucher S, Meyer F (1992) The morphological approach to segmentation: the watershed transformation. In: Dougherty E (ed) Mathematical morphology in image processing. Marcel Dekker, NY, pp 433-481

Brunet D, Sills D (2017) A generalized distance transform: Theory and applications to weather analysis and forecasting. IEEE Trans Geosci Remote Sens 55(3):1752-1764

Challa A, Danda S, Sagar BSD (2016) Morphological interpolation for temporal changes. In: 2016 IEEE international geoscience and remote sensing symposium (IGARSS), Beijing, pp 33583361. https://doi.org/10.1109/igarss.2016.7729868 
Chockalingam L, Sagar BSD (2003) Automatic generation of sub-watershed map from Digital Elevation Model: a morphological approach. Int J Pattern Recognit Artif Intell 17(2):269-274

Chockalingam L, Sagar BSD (2005) Morphometry of networks and non-network spaces. J Geophys Res-Solid Earth (American Geophysical Union) 110(B08203). https://doi.org/10. 1029/2005jb003641

Danda S, Challa A, Sagar BSD (2016) A morphology-based approach for cloud detection. In: 2016 IEEE international geoscience and remote sensing symposium (IGARSS), Beijing, pp 80-83. https://doi.org/10.1109/igarss.2016.7729011

Gastner MT, Newman M (2004) Diffusion-based method for producing density-equalizing maps. Proc Natl Acad Sci 101(20):7499-7504

Gusein-Zade S, Tikunov V (1993) A new technique for constructing continuous cartograms. Cartogr Geogr Inf Syst 20(3):66-85

Harbaugh JW (2014) Letter to the editor: what do mathematical geoscientists do? IAMG Newsl 89:5

Keim D, North S, Panse C (2004) Cartodraw: a fast algorithm for generating contiguous cartograms. IEEE Trans Vis Comput Graph 10:95-110

Kocmoud C (1997) Constructing continuous cartograms: a constraint-based approach, MA thesis, Texas A \& M University

Lantuejoul C (1978) La sequelettisation et son application aux mesures topologiques des mosaiques polycristallines. These de Docteur-Ingnieur. School of Mines, Paris, France

Lantuejoul C, Beucher S (1981) On the use of the geodesic metric in image analysis. J Microsc 121(1):39-49

Lim SL, Sagar BSD (2008a) Cloud field segmentation via multiscale convexity analysis. J Geophys Res-Atmos 113:D13208. https://doi.org/10.1029/2007jd009369. (17 pages)

Lim SL, Sagar BSD (2008b) Derivation of geodesic flow fields and spectrum in digital topographic basins, Discrete Dyn Nat Soc (Article ID 312870, 26 pages). https://doi.org/10. $1155 / 2008 / 312870$

Lim SL, Koo VC, Sagar BSD (2009) Computation of complexity measures of morphologically significant zones decomposed from binary fractal sets via multiscale convexity analysis. Chaos Solitons Fractals 41(3):1253-1262

Lim SL, Sagar BSD, Koo VC, Tay LT (2011) Morphological convexity measures for terrestrial basins derived from digital elevation models. Comput Geosci 37(9):1285-1294

Mandelbrot BB (1982) Fractal geometry of nature. WH Freeman \& Co, San Francisco, p 468

Maragos PA (1989) Pattern spectrum and multiscale shape representation. IEEE Trans Pattern Anal Mach Intell 11:701-716

Maragos PA, Schafer RW (1986) Morphological skeleton representation and coding of binary images. In: IEEE transactions on acoustics, speech and signal processing, ASSP-34(5)

Matheron G (1975) Random sets and integral geometry. Wiley, New York

Meyer F (1980) Feature extraction by mathematical morphology in the field of quantitative cytology. Technical report of Ecole nationale superiere des mines de Paris, Fountainbleau, France

Miliaresis GC, Argialas DP (1999) Segmentation of physiographic features from global digital elevation model/GTOPO30. Comput Geosci 25:715-728

Najman L, Talbot H (2010) Mathematical morphology. Wiley, p 507

Najman L, Barrera J, Sagar BSD, Maragos P, Schonfeld D (2012) Preface on filtering and segmentation with mathematical morphology. IEEE J Sel Top Signal Process 6(7):736-886

Radhakrishnan P, Sagar BSD, Teo LL (2004) Estimation of fractal dimension through morphological decomposition. Chaos Solitons Fractals 21(3):563-572

Rajashekara HM, Vardhan P, Sagar BSD (2012) Generation of zonal map from point data via weighted skeletonization by influence zone. IEEE Geosci Remote Sens Lett 9(3):403-407

Rivest JF, Beucher S, Delhomme JP (1992) Marker-controlled segmentation: an application to electrical borehole. J Electron Imaging 1(2):136-142

Sagar BSD (1996) Fractal relations of a morphological skeleton. Chaos Solitons Fractals 7 (11):1871-1879 
Sagar BSD (1998) Numerical simulations through first order nonlinear difference equation to study highly ductile symmetric fold (HDSF) dynamics: a conceptual study. Discret Dyn Nat Soc 2 (4):281-298

Sagar BSD (1999a) Estimation of number-area-frequency dimensions of surface water bodies. Int J Remote Sens 20(13):2491-2496

Sagar BSD (1999b) Morphological evolution of a pyramidal sandpile through bifurcation theory: a qualitative model. Chaos Solitons Fractals 10(9):1559-1566

Sagar BSD (2000a) Fractal relation of medial axis length to the water body area. Discret Dyn Nat Soc 4(1):97

Sagar BSD (2000b) Multi-fractal-interslipface angle curves of a morphologically simulated sand dune. Discret Dyn Nat Soc 5(2):71-74

Sagar BSD (2001a) Generation of self organized critical connectivity network map (SOCCNM) of randomly situated surface water bodies, Letters to Editor. Discret Dyn Nat Soc 6(3):225-228

Sagar BSD (2001b) Hypothetical laws while dealing with effect by cause in discrete space, Letter to the Editor. Discret Dyn Nat Soc 6(1):67-68

Sagar BSD (2001c) Quantitative spatial analysis of randomly situated surface water bodies through f- $\alpha$ spectra. Discret Dyn Nat Soc 6(3):213-217

Sagar BSD (ed) (2001) For a special issue of Journal of Mathematical Geosciences. In: Memory of the Late Professor SVLN Rao, vol 33, no 3. Kluwer Academic Publishers pp 245-396

Sagar BSD (2005a) Discrete simulations of spatio-temporal dynamics of small water bodies under varied streamflow discharges, (invited paper). Nonlinear Process Geophys (American Geophysical Union) 12:31-40

Sagar BSD (2005b) Qualitative models of certain discrete natural features of drainage environment. Allied Publishers Limited, New Delhi, p 232

Sagar BSD (2007) Universal scaling laws in surface water bodies and their zones of influence. Water Resour Res 43(2):W02416

Sagar BSD (ed) (2009) In: Proceedings on "spatial information retrieval, analysis, reasoning and modelling" seminar held during 18-20 Mar 2009, p 231

Sagar BSD (2010) Visualization of spatiotemporal behavior of discrete maps via generation of recursive median elements. IEEE Trans Pattern Anal Mach Intell 32(2):378-384

Sagar BSD (2013) Mathematical morphology in geomorphology and GISci. CRC Press, Boca Raton. p 546

Sagar BSD (2014a) Cartograms via mathematical morphology. Inf Vis 13(1):42-58

Sagar BSD (2014b) Erratum to visualization of spatiotemporal behavior of discrete maps via generation of recursive median elements. In: IEEE Trans Pattern Anal Mach Intell vol 36(3)

Sagar BSD (2018) Variable-specific classification of zones, pairs of zones, and clusters of a spatial system via modified gravity model. In: IEEE Trans Emerging Topics Comput (In Press). https://doi.org/10.1109/tetc.2016.2633436

Sagar BSD, Bruce LM (Eds.) (2005) Surficial mapping. IEEE Geosci Remote Sens Lett 2(4): 375-408

Sagar BSD, Chockalingam L (2004) Fractal dimension of non-network space of a catchment basin Geophys. Res. Lett (American Geophysical Union) 31(12):L12502

Sagar BSD, Lim SL (2015a) Ranks for pairs of spatial fields via metric based on grayscale morphological distances. IEEE Trans Image Process 24(3):908-918

Sagar BSD, Lim SL (2015b) Morphing of grayscale DEMs via morphological interpolations. IEEE J Sel Top Appl Earth Observ Remote Sens 8(11):5190-5198

Sagar BSD, Murthy KSR (2000) Generation of fractal landscape using nonlinear mathematical morphological transformations. Fractals 8(3):267-272

Sagar BSD, Rao BSP (1995a) Computation of strength of nonlinearity in Lakes, letter to the editor. Comput Geosci 21(3):445

Sagar BSD, Rao BSP (1995b) Fractal relation on perimeter to the water body area. Curr Sci 68(11):1129-1130

Sagar BSD, Rao BSP (1995c) Possibility on usage of return maps to study dynamics of lakes: hypothetical approach. Curr Sci 68(9):950-954 
Sagar BSD, Rao BSP (1995d) Ranking of Lakes: Logistic maps. Int J Remote Sens 16(2):368-371

Sagar BSD, Rao CB (eds) (2003) Special issue on quantitative image morphology. Int J Pattern Recogn Artif Intell 17(2):163-330

Sagar BSD, Serra J (eds) (2010) Special Issue on "spatial information retrieval, analysis, reasoning and modelling". Int J Remote Sens 31(22):5747-6032

Sagar BSD, Tien TL (2004) Allometric power-law relationships in a Hortonian Fractal DEM. Geophys Res Lett (American Geophysical Union), 31(6):L06501

Sagar BSD, Venu M (2001) Phase space maps of a simulated sand dune: a scope. Discret Dyn Nat Soc 6(1):63-65

Sagar BSD, Gandhi G, Rao BSP (1995a) Applications of mathematical morphology on water body studies. Int J Remote Sens 16(8):1495-1502

Sagar BSD, Venu M, Rao BSP (1995b) Distributions of surface water bodies. Int J Remote Sens 16(16):3059-3067

Sagar BSD, Omoregie C, Rao BSP (1998a) Morphometric relations of fractal-skeletal based channel network model. Discret Dyn Nat Soc 2(2):77-92

Sagar BSD, Venu M, Gandhi G, Srinivas D (1998b) Morphological description and interrelationship between force and structure: a scope to geomorphic evolution process modelling. Int J Remote Sens 19(7):1341-1358

Sagar BSD, Venu M, Murthy KSR (1999) Do skeletal network derived from water bodies follow Horton's laws? J Math Geol 31(2):143-154

Sagar BSD, Venu M, Srinivas D (2000) Morphological operators to extract channel networks from digital elevation models. Int J Remote Sens 21(1):21-30

Sagar BSD, Srinivas D, Rao BSP (2001) Fractal skeletal based channel networks in a triangular initiator basin. Fractals 9(4):429-437

Sagar BSD, Rao CB, Raj B (2002) Is the spatial organization of larger water bodies heterogeneous? Int J Remote Sens 23(3):503-509

Sagar BSD, Murthy MBR, Radhakrishnan P (2003a) Avalanches in numerically simulated sand dune dynamics. Fractals 11(2):183-193

Sagar BSD, Murthy MBR, Rao CB, Raj B (2003b) Morphological approach to extract ridge-valley connectivity networks from digital elevation models (DEMs). Int J Remote Sens 24(3): $573-581$

Sagar BSD, Rangarajan G, Veneziano D (eds) (2004) Special issue on fractals in geophysics. Chaos Solitons \& Fractals 19(2):237-462

Sagar BSD, Rajesh N, Vardhan SA, Vardhan P (2013) Metric based on morphological dilation for the detection of spatially significant zones. IEEE Geosci Remote Sens Lett 10(3):500-504

Sathymoorthy D, Radhakrishnan P, Sagar BSD (2007) Morphological segmentation of physiographic features from DEM. Int J Remote Sens 28(15)

Serra J (1982) Image analysis and mathematical morphology. Academic Press, London, p 610

Serra J (1988) Image analysis and mathematical morphology: theoretical advances. Academic Press, p 411

Soille P (2003) Morphological image analysis, principles and applications, 2nd edn. Springer, Berlin

Sternberg SR (1986) Greyscale morphology. Comput Vis Graph Image Process 35:333-355

Tay LT, Sagar BSD, Chua HT (2005a) Derivation of terrain roughness indicators via Granulometries. Int J Remote Sens 26(18):3901-3910

Tay LT, Sagar BSD, Chuah HT (2005b) Allometric relationships between travel-time channel networks, convex hulls, and convexity measures. Water Resour Res (American Geophysical Union) 46(2):W06502. https://doi.org/10.1029/2005WR004092

Tay LT, Sagar BSD, Chuah HT (2005c) Analysis of geophysical networks derived from multiscale digital elevation models: a morphological approach. IEEE Geosci Remote Sens Lett 2(4): 399-403

Tay LT, Sagar BSD, Chuah HT (2007) Granulometric analysis of basin-wise DEMs: a comparative study. Int J Remote Sens 28(15):3363-3378 
Teo LL, Sagar BSD (2005) Reconstruction of pore space from pore connectivity network via morphological transformations. J Microsc (Oxford) 219(Pt 2):76-85

Teo LL, Sagar BSD (2006) Modeling, characterization of pore-channel, throat and body. Discret Dyn Nat Soc (an International Journal) (Article ID 89280) 1-24

Teo LL, Radhakrishnan P, Sagar BSD (2004) Morphological decomposition of sandstone pore-space: fractal power-laws. Chaos Solitons \& Fractals

Vardhan SA, Sagar BSD, Rajesh N, Rajashekara HM (2013) Automatic detection of orientation of mapped units via directional granulometric analysis. IEEE Geosci Remote Sens Lett 10(6):1449-1453

Open Access This chapter is licensed under the terms of the Creative Commons Attribution 4.0 International License (http://creativecommons.org/licenses/by/4.0/), which permits use, sharing, adaptation, distribution and reproduction in any medium or format, as long as you give appropriate credit to the original author(s) and the source, provide a link to the Creative Commons license and indicate if changes were made.

The images or other third party material in this chapter are included in the chapter's Creative Commons license, unless indicated otherwise in a credit line to the material. If material is not included in the chapter's Creative Commons license and your intended use is not permitted by statutory regulation or exceeds the permitted use, you will need to obtain permission directly from the copyright holder. 